\title{
Aha! I know where I am: The contribution of visuospatial cues to reorientation in urban environments
}

\begin{abstract}
Reorientation depends greatly on the perceived geometric information, which constantly changes during navigation in urban environments. Environmental novelty, as a driver of exploratory behavior, is likely to engender this spatial Aha! moment. The paper investigates the contribution of two qualitatively different types of novelty, corresponding to distinct visuospatial cues: (a) situations that cause surprise e.g. a sudden change in spaciousness; versus (b) situations that engender mystery e.g. a change in the complexity of visuospatial information and the promise of gaining new information. Visibility graph analysis is used to quantify and examine these hypotheses in relation to participants' exploratory behavior and brain dynamics (EEG) during virtual navigation. The findings suggest that reorientation is a spatial boundary effect, associated primarily with a change in visuospatial complexity.
\end{abstract}

Keywords: reorientation; Aha! Moment; virtual navigation; EEG; visibility graph analysis.

\section{Introduction}

A sudden and spontaneous transition from the state of being disoriented and lost into the state of being situated in space and oriented is a common Aha! experience had by many people whilst wayfinding. When, for example, we are being passively displaced (e.g. traveling on the underground) spatial updating is disrupted (Julian et al., 2018) and our sense of direction might not be anchored to the external environment. We may start walking confidently towards a certain direction only to realize suddenly that we are heading away from our goal. This moment of reorientation involves two key subcomponents: the sudden self-localization, which requires context retrieval (the recovery and reorientation to a new frame of reference ), and the retrieval of relevant 
heading information ${ }^{1}$ (Julian et al., 2018; Julian et al., 2015). The focus of the paper is on the first component of self-localization (Barry \& Burgess, 2014; Jeffery, 2007), and the visuospatial conditions that contribute to this Aha! experience.

The experience of disorientation and sudden, spontaneous reorientation is often associated with "a gut feeling" (Montello, 2020) which suggests that this experience is qualitatively comparable to the Aha! moment experienced during insightful thinking. Insightful thinking refers to the process that leads to insights, a new sudden understanding that emerges into conscious awareness with a sudden abruptness. It involves the reorganization of the elements of the mental representation, a transformative step to overcome the mental impasses or conflict created by the initial incomplete or misleading representation (Shen et al., 2013). In contrast, during deliberate analytical thinking, there is a conscious and incremental awareness of the solution (Kounios \& Beeman, 2014). Early research on insightful thinking reported the involvement of the right hippocampus, which suggests that reorientation of one's

${ }^{1}$ This distinction is illustrated well in one of the subjective reports on the experience of disorientation, collected by psychologist Alfred Binet (1894). The individual here describes how sudden place/context recognition and retrieval preceded heading retrieval, which finally "destroyed the illusion" of misorientation:

“...instead of taking the right to return to the Place de la Republique, I took the left toward the Hotel de Ville. How did I realize my mistake? ... It is certain that while on my way I felt sure of meeting the Place de la Republique. Thus, my confusion was extreme on coming to the Hotel de Ville. As before, I was some moments in recognizing it. Then I recognized the Hotel de Ville, without destroying the illusion. It disappeared, however, very quickly, and, I think, like the others, when I understood the cause of my mistake." (Observation II, p. 341, Binet, 1894) 
thinking during creative insights might be subserved by a similar hippocampal function to that of reorientation during navigation (Luo \& Niki, 2003).

The subjective experience of disorientation has recently received increased attention regarding its affective and metacognitive dimensions and the need to differentiate among moments in the time course of such episodes (Fernández Velasco \& Casati, 2020a, 2020b; Montello, 2020). Core components of creative insights (Knoblich et al., 1999; Sandkuhler \& Bhattacharya, 2008; Shen et al., 2013; Shen et al., 2017; Weisberg, 2013) can be also identified during the time course of reorientation. Firstly, there is a period of mental impasses when the initial representation is incomplete or misleading (not knowing where one is and/or their heading direction). The confusion and uncertainty that arises (Montello, 2009, 2020) during disorientation, when "one has no sense of direction at all" (Binet, 1894) or when the "the spatial representation itself becomes unstable" (Dudchenko, 2010), or after misorientation, when there is a misalignment between the reference frame of the current mental spatial representation and the external environment (Binet, 1894; Dudchenko, 2010; Montello, 2020), is followed by conflict resolution processes ${ }^{2}$. Conflict resolution processes, triggered by uncertainty during disorientation, often result in a perceptual restructuring, a sudden representational switch, and the formation of novel associations. This leads to a deeper understanding of one's position and heading direction and a sudden (Aha!) experience of reorientation.

\footnotetext{
${ }^{2}$ Another interesting point from Observation II in Binet's article refers to the occurrence of such processes: "At the end of a certain period of conflict, three or four minutes perhaps, the illusion disappeared. And when it disappeared for one street or one object, it disappeared for all. I could then immediately take the road and direct myself correctly" (Observation II, p. 341, Binet, 1894).
} 
The representational switch is a crucial component of the Aha! moment.

According to Arthur Koestler's theory of the 'bisociation of matrices', the Aha! moment involves "the perceiving of a situation...in two self-consistent but habitually incompatible frames of reference" (Koestler, 1964, p. 35). Similarly, the experience of reorientation, as described in great detail by Brian Massumi (1999, pp. 179-183), appears to involve the sudden simultaneous experience of two different frames of reference: the "proprioceptive system of self-reference" and the "exoreferential singlesense functioning" associated with form in configuration. The sudden 'blending' of these reference frames forms a third hinge-dimension of experience ${ }^{3}$, an instance, perhaps, of the well-known phenomenon of the Aha! moment.

Another instance of the Aha! moment in spatial learning is when there is a sudden integration of distinct and self-consistent knowledge chunks, that is, during the spontaneous understanding of the relationship between places learned during different travel experiences. According to Montello's (1998) new framework of spatial microgenesis this moment is considered as a significant and sophisticated step in spatial learning, which results in a qualitative shift in spatial knowledge. ${ }^{4}$

${ }^{3}$ According to Massumi (1999) the hinge-dimension of experience emerges through cross-sense referencing as, for example, in the case of synaesthesia where this form of experience is manifested perceptually (e.g. sounds seen as colors). During reorientation, cross-sense referencing occurs when "vision's conscious forms-in-configuration feed back into the vectorial tendency-plus-habit of proprioception, and where proprioception feeds forward into vision". He notes that this is a hinge-dimension between quantitative and qualitative space and "where we go to find ourselves when we are lost is where the senses fold into and out of each. We always find ourselves in this fold in experience" (Massumi, 1999, p. 182).

${ }^{4}$ In contrast to the dominant framework of spatial knowledge acquisition, which supports the idea of transitions between discrete and qualitative different stages of spatial knowledge (i.e. landmark knowledge, route knowledge and survey knowledge), Montello proposes a 
The translation of spatial information (or sudden bisociation) between different systems of spatial representation e.g. egocentric and allocentric reference frames (Burgess, 2006; Mou et al., 2006) is facilitated by the retrosplenial cortex (Alexander \& Nitz, 2015; Byrne et al., 2007; Vann et al., 2009). The retrosplenial cortex, involved in the process of self-localization and orientation, along with the parahippocampal place area, associated with place recognition, are thought to play an important role during reorientation and enable anchoring the cognitive map to stable elements of the environment such as environmental geometry and shape (Chadwick \& Spiers, 2014; Epstein et al., 2017; Julian et al., 2018; Marchette et al., 2014).

\subsection{The Role of Environmental Geometry}

Findings from behavioral and cognitive neuroscience studies suggest that reorientation depends greatly on the geometry ${ }^{5}$ of the environmental boundaries (Cheng \& Newcombe, 2005; Hermer \& Spelke, 1994; Keinath et al., 2017; S. A. Lee \& E. S. Spelke, 2010). Boundary geometry plays a significant role in spatial memory (Bellmund et al., 2019; Hartley et al., 2004) and in orienting the hippocampal map of mice after disorientation (Keinath et al., 2017). Changes in the environmental shape induce hippocampal remapping (Fyhn et al., 2007; Lever et al., 2002; Wills et al., 2005), which may reflect the formation and recruitment of distinct cognitive maps (spatial or

new framework for understanding spatial microgenesis where the only qualitative shift during spatial knowledge acquisition is the integration of separate learned spaces (Montello, 1998).

${ }^{5}$ There is, however, an ongoing debate (Cheng, Huttenlocher, \& Newcombe, 2013; Duval, 2019) between theories supporting the geometric-module framework of reorientation (Cheng, 1986; Gallistel, 1990) versus a view-matching mechanism(e.g. Nardini, Thomas, Knowland, Braddick, \& Atkinson, 2009; Stürzl, Cheung, Cheng, \& Zeil, 2008). 
conceptual) for different navigational or behavioral contexts (Bellmund et al., 2018; Julian et al., 2018).

Disoriented rats and young children reorient themselves using primarily the geometry of the environment (Cheng, 1986; Hermer \& Spelke, 1994, 1996; Lee \& Spelke, 2011) despite the presence of salient non-geometric cues. The contribution of non-geometric features in reorientation, (e.g. visual cues or single discrete landmarks) is still a matter of debate (Julian et al., 2018) as well as their relation to linguistic processes and the development of spatial language (Hupbach et al., 2007). Julian and colleagues (2018), for example, argue that discrete landmark-objects are useful if they have been previously experienced as being stable while in an oriented state while the environmental shape and boundary geometry provide stable frames of reference for reorientation.

Relevant studies have highlighted the importance of various and different geometric components (Lee et al., 2012; Lew et al., 2010; Newcombe et al., 2010; Sturz et al., 2011) and it is still not entirely clear what sort of the geometric or environmental properties are used as cues for reorientation (Cheng et al., 2013; Julian et al., 2018; S. Lee \& E. Spelke, 2010). Different geometric cues may play different roles in the spatial representation (Jeffery, 2010) such as determining the global shape and principal axes (Gallistel, 1990), providing detailed localized information enabling the computation of shape parameters (Duval, 2019) and/or acting as contextual cues, indicating an environmental change ${ }^{6}$, which, according to Jeffery (2010), has been relatively understudied in behavioral experiments. Large environmental change as opposed to

\footnotetext{
${ }^{6}$ Interestingly, findings from a change detection task of visual shape (Barenholtz, Cohen, Feldman, \& Singh, 2003) suggest that changes that increase the concavity of the shape are more salient than changes that increase the convexity of the shape.
} 
small changes, for example, cause remapping of grid cells (translation and/or rotation of their fields in response to environmental change) and remapping also occurs in place cells following large changes to the environment (Jeffery, 2011; Wills et al., 2005). Furthermore, the effect of geometry on reorientation is usually investigated in enclosed spaces rather than complex urban environments (Wolbers \& Wiener, 2014). To address this research gap, the study is focused on environmental changes and examines what sort of formal visuospatial properties may engender the Aha! moment of reorientation in large-scale urban environments.

\subsection{Environmental Change and Novelty}

During movement in large-scale environments, where the perceived visuospatial information is constantly changing, novelty and sudden environmental change have a strong influence on exploratory behavior. Detection of novelty and unexpected events plays an important role in incidental learning and gaining insights into an implicit regularity within a sequence of events (Haider \& Rose, 2007; Rünger \& Frensch, 2008), and is associated with increased hippocampal activity during wayfinding (Kaplan et al., 2014; Kumaran \& Maguire, 2006). However, different forms of novelty are associated with different patterns of brain activity in the medial temporal lobe (Kaplan et al., 2014; Köhler et al., 2005; Strange et al., 2005). And it is possible that not all novel stimuli act as determinants of exploratory behavior (Berlyne, 1950, 1970) and may evoke different affective responses, e.g., surprise or curiosity and mystery.

Surprise can be triggered by unexpected events, resulting in an update of the mental representation (Donchin, 1981). Curiosity ${ }^{7}$ (Berlyne, 1960, 1966) is often

\footnotetext{
${ }^{7}$ Interestingly, curiosity induced by ambiguous visual stimuli was associated with activity in the anterior cingulate cortex (sensitive to conflict and arousal) and the relief of perceptual
} 
aroused by elements in the environment that are novel but also to some degree familiar, setting up some sort of expectation (Berlyne, 1950). Mystery ${ }^{8}$ (Kaplan, 1987) —a notion closely linked to curiosity—and complexity (Berlyne, 1970; Vitz, 1966) are both considered drivers of exploratory behavior, according to Kaplan's framework for environmental preference (Kaplan, 1987), and respond to the informational need to update and expand the cognitive map (Kaplan \& Kaplan, 1989; Kaplan, 1987; Kaplan, 1992). For instance, increases in environmental complexity, most likely reflecting affordances, cause significant changes in rats' exploratory behavior (Pisula et al., 2019). Mystery is often associated with the experience of surprise but it is rather qualitatively different; it includes the element of predictability and the promise of gaining new information (Kaplan, 1987).

A similar distinction between surprise and entropy highlights again the element of predictability and its distinct relation to hippocampal activity (Strange et al., 2005). The hippocampus is sensitive to novel, unpredictable events and their relative predictability within a sequence. For example, a study on sequential learning examined the brain's response to sequential predictability and reported the involvement of the hippocampal system, the parieto-occipital sulcus, the retrosplenial cortex and the anterior cingulate cortex (Harrison et al., 2006), brain regions that are also engaged during navigation. The sensitivity of these brain areas to predictability and the structure of events within a sequence probably facilitates memory mechanisms; remembering an item in relation to

curiosity with hippocampal activation and enhanced incidental memory (Jepma, Verdonschot, van Steenbergen, Rombouts, \& Nieuwenhuis, 2012).

${ }^{8}$ Similar to Berlyne's definition of epistemic curiosity as a "drive to know" (Berlyne, 1954, p. 187 ), mystery "allows one to generate hypotheses, directly parallel to the effect of partial information in an environment" (Kaplan, 1987, p. 27) but also suggests a continuity "between what can be seen and what is inferred" (Kaplan, 1987, p. 9). 
its neighboring items within a sequence or an event in relation to its context appears to be more efficient than remembering each item or event individually (Burgess et al., 2001).

\subsection{Hypotheses of the Present Study}

Consequently, two qualitatively different types of spatial novelty can be identified: spatial situations that can cause mere surprise during exploration, such as a sudden change in spaciousness when passing from a small street to a big square, and spatial situations that engender mystery such as a change in the complexity of the visual information reflecting a change in affordances and the promise of gaining new information.

We translated these spatial qualities into quantitative descriptors, namely the change in isovist area (for the change in spaciousness) and the clustering coefficient (for the change in complexity and mystery), using the space syntax technique of visibility graph analysis (see Materials and Methods for more details). Findings from recent studies suggest that space syntax measures reliably capture environmental properties that are associated, for example, with differences in performance on spatial judgment using spatial information stored in and retrieved from memory (Pagkratidou et al., 2020) and with differences in hippocampal activity (Javadi et al., 2017).

The formal descriptions of the environmental changes of spaciousness and complexity/mystery enabled testing the following hypotheses: (a) $\mathrm{H}_{\text {null }}$ : The spatial Aha! experience occurs at random locations; (b) $\mathrm{H}_{\text {spaciousness: The spatial Aha! }}$ experience is more likely to occur at locations where suddenly a large amount of visual information becomes available, as measured by a change in isovist area; and (c)

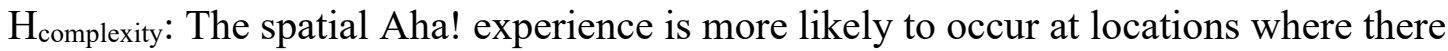
is a sudden change in the complexity of the visual information reflecting mystery and 
the promise of gaining more information, as measured by the isovist clustering coefficient.

The ecological validity of the study was important to us, so we developed an experimental design that was as close as possible to the real-world scenario of "coming out of the underground station' (i.e. teleportation), which is often followed by a sudden experience of reorientation. Data analyses of participants' exploratory behavior and EEG recordings were focused on sudden changes of the visuospatial information preceding participants' response (stimulus-locked analysis) and on participants' differences in brain dynamics just before the response (response-locked analysis).

Relevant empirical studies suggest that successful self-localization during reorientation it is likely to be associated with a decrease in alpha power $(8-13 \mathrm{~Hz})$ at posterior areas and a frontocentral theta $(4-7 \mathrm{~Hz})$ increase. Decreases in alpha power in or near retrosplenial cortex were interpreted as reflecting the integration of information from the visual flow with a representation of self-location (Lin et al., 2015) and the retrieval of information from allocentric representations (Chiu et al., 2012). Posterior alpha decrease was also observed before spontaneous backtracking (Javadi et al., 2019); a behavioral change in the direction of movement that shortens the path towards the goal and is often observed during reorientation. Increases in frontocentral theta activity were associated to the sudden availability of a shorter path, a shortcut, likely reflecting conflict monitoring related processes (Patai et al., 2019) . Theta power increase,, generated in or near the anterior cingulate cortex, was also linked to increases in spatial working memory demands to update information regarding position and orientation (Lin et al., 2015). Based on these findings and considering that backtracking is a behavior often observed after reorientation and that finding a shortcut requires understanding of a new trajectory between locations or separately learned places, we 
expect that the spatial Aha! moment of reorientation will be accompanied by the following response-related brain dynamics: a parietal alpha decrease (channels P7 \& P8) and a frontocentral theta increase (channels FC5 \& FC6).

The stimulus-related analysis is focused on the transitions of the perceived visuospatial information during movement and how these sensory transitions may contribute to the moment of reorientation. Extensive theoretical and empirical work on movement-related and memory-related oscillations (for a comprehensive review see Ekstrom \& Watrous, 2014) is focused on human hippocampal theta modulations, which tend to manifest at lower frequencies during virtual navigation (Bohbot et al., 2017; Jacobs, 2014; Watrous et al., 2011). In studies using intracranial EEG, low frequency oscillations are often linked to spatial updating processes and encoding of translational movement (Bush et al., 2017; Kaplan et al., 2012) but might also be related to visual input from optic flow and spatial processing (Watrous et al., 2011). Relevant noninvasive EEG/MEG studies have reported increase theta activity over the frontal regions (Araújo et al., 2002; Bischof \& Boulanger, 2003; Jaiswal et al., 2010; Kaplan et al., 2017). The relationship between the frontal EEG response and hippocampal theta has been explored but still remains unclear (Long et al., 2014; Mitchell et al., 2008).

Interestingly, some EEG studies on memory and navigation have reported a peak EEG power near or below 4Hz (Araújo et al., 2002; Kaplan et al., 2012; Staudigl \& Hanslmayr, 2013), which falls at the limit of the conventional 4-7Hz frequency range. More recent relevant studies are focused on a delta/theta frequency range (e.g. 2$7 \mathrm{~Hz}, 2-8 \mathrm{~Hz})$ and reported increase delta/theta activity related to movement, spatial exploration, spatial learning and memory (Liang et al., 2018; Long et al., 2014; Snider et al., 2013; Spaak \& de Lange, 2020). Adopting a similar approach, we focused on the delta/theta frequency $(2-7 \mathrm{~Hz})$ to examine how changes in visuospatial information, 
within a $3 \mathrm{~s}$ time window preceding participants response (contributing to the moment of reorientation), are linked to differences in EEG power over frontal regions 9 .

\section{Materials and Methods}

\subsection{Participants and Recruitment Method}

Data was collected from twenty participants ( 9 females), a comparable sample size with recent relevant EEG studies (e.g. Chiu et al., 2012; Lin et al., 2015; Patai et al., 2019). Participants (mean age:33.8 years, range: 20 - 43years) were right-handed with normal or corrected-to-normal vision, and none reported any history of psychiatric or neurological problems. Half of the participants had a background in a discipline related to the built environment (e.g. architects, geographers). Participants' perceptions of their spatial abilities were assessed using the Santa Barbara Sense of Direction (SBSOD) test (Hegarty et al., 2002) with a scale from 1 to $7(M=4.67, S E=0.23)$. Two participants were recruited from the PALS Divisional Subject Pool in exchange for course credit and the rest responded to email advertisement and participated voluntarily. The research was approved by the ethics committee at University College London (Project ID 6359/002) and all participants gave their informed consent.

\subsection{Data Acquisition}

Scalp EEG activity was recorded using the Emotiv headset, a wireless EEG system with 14 electrodes, as shown in Figure 2, which can be reliably used for scientific research (Melnik et al., 2017; Pietto et al., 2018). The raw EEG data was collected via a

\footnotetext{
${ }^{9}$ To better capture the frontal EEG power and because the Emotiv does not have the Fz channel we included four channels of the frontal region, namely F3, F4, FC5, FC6.
} 
Bluetooth USB chip and saved using the Emotiv Testbench software. A desktop VR setup was used for this experiment. This allowed all participants to complete the tasks without experiencing any motion sickness, which is often observed when using VR headsets. The same computer was used for the virtual navigation and EEG recordings to have more accurate timing of the event-markers (e.g. keystrokes). During each trial, participants' position and heading direction, were recorded into a log file every 0.25 seconds.
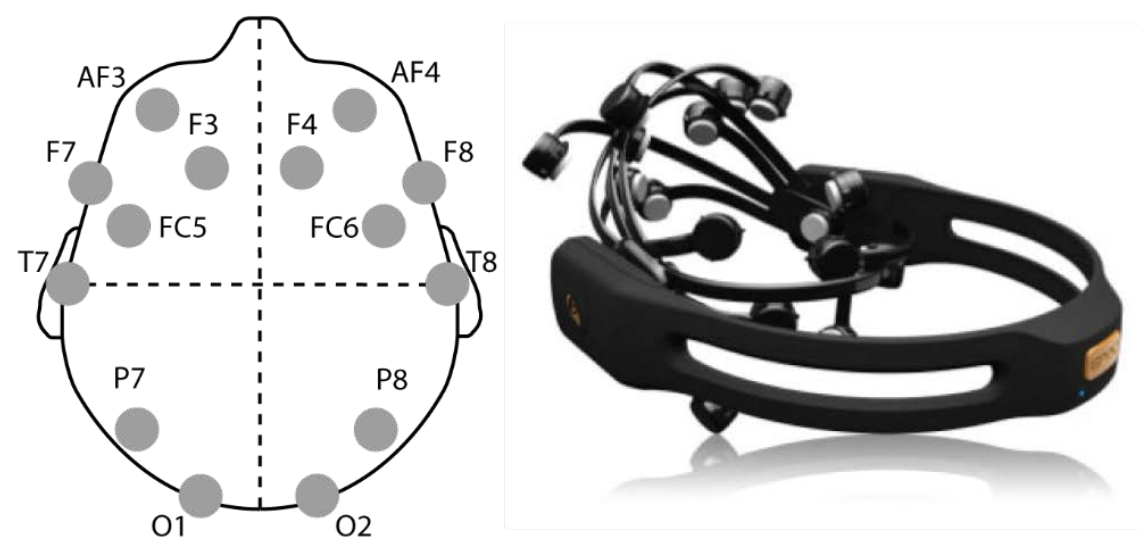

Figure 1. Emotiv EPOCH 14-channels location

\subsection{The Virtual Environment}

The virtual reality urban environments were created and developed in the game engine Unity3D (version 5.3.4). The three-dimensional models, created in Rhino3D, represent real urban regions from three European cities: a neighborhood in central Athens (Psirri and Monastiraki tube station), an area in the center of Barcelona (area of El Raval and Sant Antoni) and a region in central London (around Tottenham Court Road tube station). These are central, commercial, and dense urban environments of metropolitan areas, planned around mass public transport stations. The three urban regions have comparable spatial configurations and similar characteristics in terms of streets networks, urban grids, building density and open spaces (Figure 2A). 
More specifically, the three environments have major street axes that provide visual access and mark out district subregions. Our assumption is that the identification of distinct subregions facilitates a reduction in the perceived layout complexity because clustering urban elements into separate units reduces the information load (e.g the number of disparate components). In most of these subregions there are open spaces with different sizes, shapes and/or orientation. In addition, in each environments there are at least two distinct urban grids that help differentiate further the subregions in terms of grid density, orientation and/or type (orthogonal and organic).Visual inspection of the map of each environment permits the individual to identify the particular patterns of street networks and the relative location between the different spatial elements. We have chosen these urban regions because we think that these characteristics can be easily identified when studying the map of each environment and provide enough information for participants to be able to orient themselves during navigation.

The 3D models of the virtual environments replicate the volumetric information of the actual urban environments (e.g. actual heights of buildings and street configurations). However, the visual characteristics of the buildings are simplified and the virtual environments include only random buildings with brick textures and grey colored buildings, as shown in Figure 2B. To prevent participants from orientating based on the direction of light, the buildings do not cast any shadows on other buildings or streets and there is no visible sun that could act as an orientation cue. Another possible global cue often used for reorientation are patterns of cloud in the sky. Although it is possible that larger parts of the sky are visible during certain moments, there are no salient characteristic patterns in the image used for the sky in the VR environment and an individual would need to study extensively the image of the sky in order to be able to use clouds as global cues. 
A.

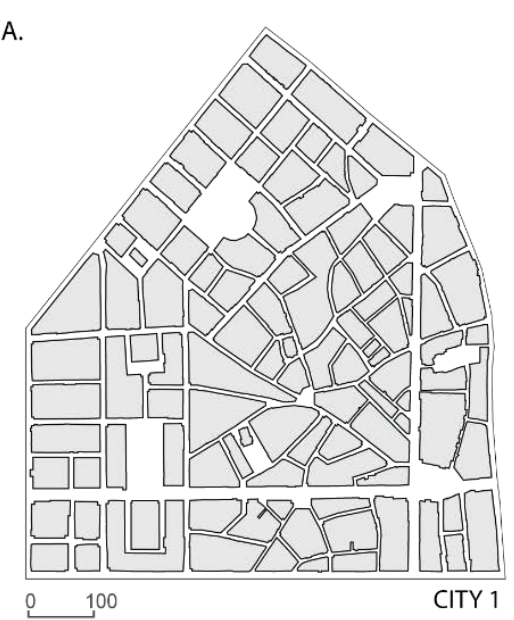

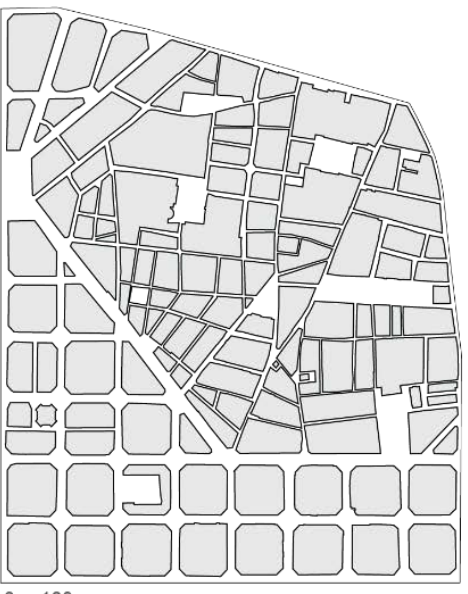

CITY 2

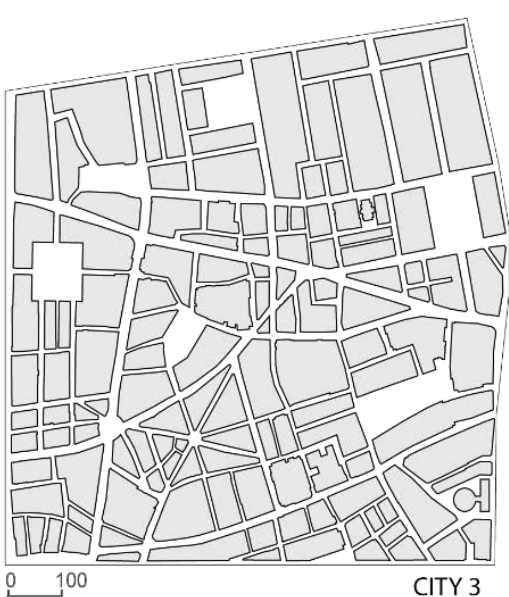

CITY 3

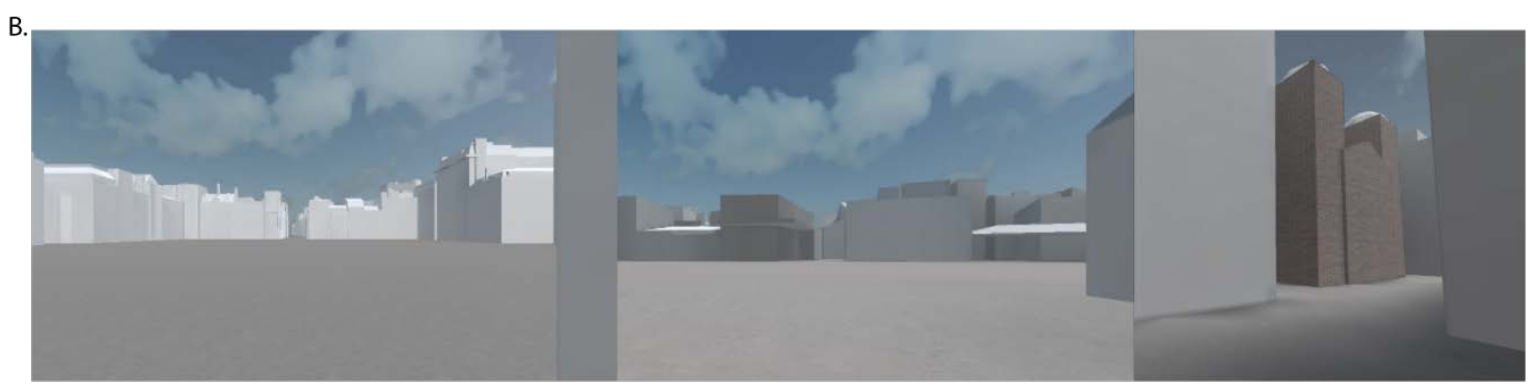

Figure 2. A. Maps of the virtual reality environments; B. First-person perspective views of the three virtual reality environments.

\subsection{Experimental Procedure}

Participants were first verbally informed about the structure of the experiment and the task. Because movement causes noise artifacts to the EEG data, participants were asked to stay as still as possible during the experiment. Although think-aloud protocols could have been revealing, especially in identifying the Aha! moment, jaw movements cause great artifacts and, thus, participants were instructed to avoid speaking during the recordings. Data regarding spatial abilities and properties of the individuals (age, gender, subject of studies, SBSOD scores etc.) were collected at the end of the experiment.

Participant were verbally informed about the following rationale of the experimental task: Imagine you are a tourist in a new city. After studying the map of the 
city you are visiting, you start exploring the city but end up being lost. Not having enough battery on your mobile phone, you will probably switch on the map application once you arrive at a place where you suddenly experience a feeling that in this location it is more likely to reorient.

Participants were first familiarized with the instructions and procedure in a simple urban training environment with a grid of $4 \times 5$ building blocks. To navigate the urban environment participants were instructed to use the navigation keys (e.g., arrows for up, down, left, right). Speed was fixed when the key with the arrow indicating up was pressed, and participants could release it to pause movement. Each participant was asked to complete 8 trials in each of the three environments, giving a total of 24 trials for analysis per individual ( 8 trials $\mathrm{x} 3$ cities). The three environments were not randomized because the experimental set up was not designed to test for differences between the environments. Before the start of each block of trials within the same environment the instructions were presented on the computer screen. After reading the instructions, participants were able to proceed by pressing the spacebar on the keyboard. Each block of trials of the experiment was divided into two stages, as shown in Figure 3, comprising a study and a navigation phase:

a) Study phase: At the beginning of each session a map of the city region was presented on the screen. This offered participants an initial survey knowledge of the spatial configuration. Participants were asked to carefully study the map and focus on the spatial layout. The study map phase did not have a time limit and participants were asked to press the 'ready' button as soon as they were ready to proceed to the next phase.

b) Navigation phase: Each trial began from a different location (random order), placing the participant in an initial state of disorientation. Participants' task was to 
explore the environment until they had the feeling of knowing their location in space and were thus able to orient themselves in relation to the map presented in the study phase. At this point, they were instructed to press the spacebar on the keyboard to give their response.

Pressing the spacebar activated a graphic user interface (GUI), shown in Figure 3. The GUI included an image of the map of the environment, a slider, a 'save response' button and an 'exit' button. Subjects were asked to rotate the map on the screen, using the mouse, and align it with their heading direction. They were asked to rate their confidence regarding their response using the slider as certain (3), probably right (2) or not sure (1). Then, they had to save their response within 40 seconds, otherwise, the GUI would disappear (this was an instruction given at the start of the study). At the end of each session, the percentage of correct responses over all responses appeared on the screen. Incorrect responses included those with an absolute angular deviation of 30 degrees, following the approach adopted in similar studies focusing on alignment effects (Meilinger et al., 2014). The final number of trials included in the analysis was 464.Trials where participants entered inside a building block because of problems with the 3D model or where problems emerged with the game engine during testing were excluded from the analysis. Thus, $3 \%$ of the trials were excluded $(16 / 480)$, which is a low percentage and thus not a significant data loss. 


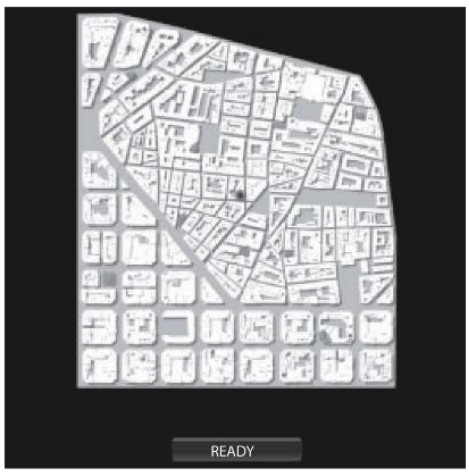

Study Map Phase

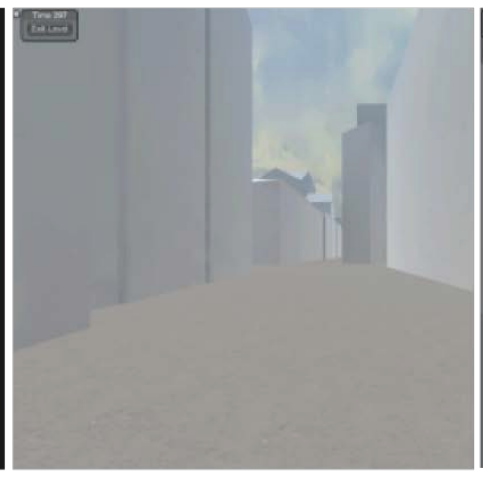

Navigation Phase

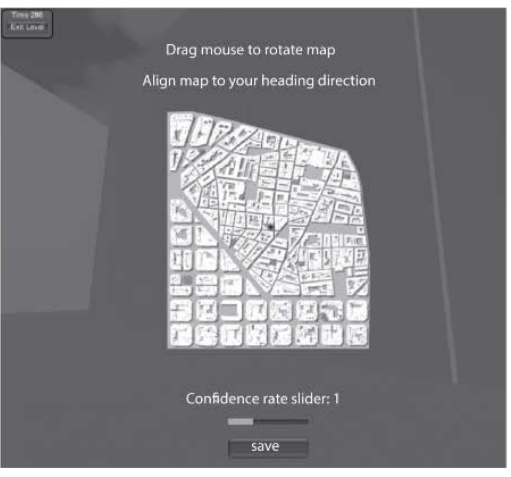

Response GUI

Figure 3. Experimental procedure (Study map phase, navigation phase, response GUI).

\subsection{Data Analyses}

\subsubsection{EEG Signal Processing}

The EEG signal can be conceptualized as a change in voltage of the electrical signal recorded from the human scalp, which is the result of firing activity of a large population of neurons and can be processed in the time-domain or the time-frequency domain. Changes in the EEG power at different frequency bands ${ }^{10}$ - delta $(2-4 \mathrm{~Hz})$, theta (4-7Hz), alpha $(8-13 \mathrm{~Hz})$, beta $(13-30 \mathrm{~Hz})$ and gamma $(>30 \mathrm{~Hz})$ - can be investigated and compared between different conditions.

The EEG signal processing steps were performed using EEGLAB (Delorme \& Makeig, 2004), an open-source toolbox for Matlab, and custom-written scripts. The EEG signal is down-sampled by the hardware at $128 \mathrm{~Hz}$ and the device applies a notch filter at 50 $60 \mathrm{~Hz}$ to remove artifacts produced from the power line. To remove slow drift artifacts (in lieu of baseline correction) a high-pass filter with $1 \mathrm{~Hz}$ cut-off was applied.

Stereotyped artifacts such as eye-blinks and eye-movement were detected and removed

\footnotetext{
${ }^{10}$ Frequency refers to the 'speed of the oscillation' measured in hertz $(1 \mathrm{~Hz}$ is one cycle per second) and power refers to the strength of the frequency band activity.
} 
using independent component analysis (ICA 'runica' function). The EEG signal was then segmented into epochs of $3 \mathrm{~s}$ duration based on the time-locking events of interest. Relative changes in mean EEG power during the 3s epochs, and within the frequency windows of interest, were calculated by subtracting the mean EEG power of a preceding time-window of equal duration. Artifacts were detected (deflections exceeding \pm 100 $\mu \mathrm{V}$ ) and excluded from further analysis.

The time-frequency analysis was performed using a complex Morlet wavelet transformation (custom-written scripts) and the power values were log-transformed. The stimulus-locked epochs were also analyzed in the time-domain using the event-related potentials (ERP) method. A low-pass filter with a cut-off at $20 \mathrm{~Hz}$ was applied to the epoched data to remove further noise (Cohen, 2014, pp. 97-107). The EEG signal was then averaged separately for each condition, channel, and participant. The ERP waveforms were mainly used here as a data quality inspection tool.

\subsubsection{Spatial Analysis}

Space syntax’s (Hillier, 1998; Hillier \& Hanson, 1984) environmental modeling techniques (e.g. axial map analysis, visibility graph analysis, isovist analysis) have been used in various studies that examine environmental properties in relation to spatial cognition and wayfinding (Conroy Dalton et al., 2012; Li \& Klippel, 2016; Penn, 2003; Peponis et al., 1990; Zimring \& Dalton, 2003), human spatial behavior (Emo, 2014; Hölscher et al., 2012), navigation performance (Barton et al., 2014) and spatial memory (Dara-Abrams, 2006; Pagkratidou et al., 2020).

For example, isovist measures (Benedikt, 1979; Benedikt \& Burnham, 1985), which describe properties of the visible space from a given point in space, appear to correlate with individual spatial experience and behavior and can capture spatial qualities linked to environmental psychology theories (Franz \& Wiener, 2005; Franz \& 
Wiener, 2008; Montello, 2007). Moreover, the human hippocampus appears to respond differentially to different graph-theoretic space syntax measures of topological properties (Javadi et al., 2017).

To quantify the environmental changes of spaciousness and complexity we used visibility graph analysis (VGA). VGA combines the idea of isovist with measurements from graph theory to calculate the intervisibility of multiple observation points distributed in a regular grid over the whole environment (Turner et al., 2001). Visibility graph measures of the three urban regions were calculated using the Depthmap software on a three-by-three meter grid covering each environment.

\section{Formal descriptions of environmental properties.}

Complexity and mystery were linked to the VGA measure of the visual clustering coefficient, which has a direct association with wayfinding and is thought to be related to spatial learning, decision-making, and path selection during exploration (Kubat et al., 2012). The clustering coefficient describes the relative intervisibility within the neighborhood of a vertex by measuring the proportion of the actual visual connections within the neighborhood of the current vertex (the point in space that generates the isovist), in relation to the number of all possible connections ${ }^{11}$ (Turner et al., 2001). Low values of the clustering coefficient are related to the notion of mystery and the promise of gaining new information since they describe locations with multidirectional visual fields where a new 'area' of the environment may be discovered with further movement (Turner et al., 2001). It has been associated with the degree of

\footnotetext{
${ }^{11}$ In graph theory is defined as the number of edges between all the vertices in the neighborhood of the generating vertex divided by the total number of possible connections with that neighborhood size (Turner et al., 2001).
} 
visual stability and the spatial quality of complexity (Franz \& Wiener, 2008) since it indicates variations in visual information and changes in the perceived environmental shape during navigation, capturing the multidirectionality ('spikiness') or convexity of the isovist shape.

Spaciousness is associated with the measure of isovist area (or neighborhood size) since rated spaciousness was found to have a high correlation with isovist area (Franz \& Wiener, 2008; Stamps, 2008, 2011). Isovist area is a geometric property of the isovist and refers to the area of space visible from a given point in space. A sudden rise in the values of area corresponds to a sudden revelation of a large amount of visual information, which can presumably cause a surprise. In addition, data from participants' exploratory behavior during virtual navigation (Conroy, 2001) suggests that locations where participants choose to pause to re-evaluate the environmental information, correlate with high values of isovist area.

Therefore, the two local VGA metrics (Figure 4) that are of interest here are isovist area, which we have used to calculate the change in isovist area, and the clustering coefficient. Although the measures are strongly anti-correlated, they are not reciprocal versions of one another, and we are interested (in our non-null hypotheses) in the more detailed ways in which they differ. The clustering coefficient captures the 'spikeness' of the geometric/environmental shape while the change in isovist area refers to the change in the area of the geometric shape. One of the clearest differences, for example, is when walking through a large space, like a broad avenue or a square: isovist area does not change much as you pass the axis of the little streets that enter in to it, but clustering coefficient does. 

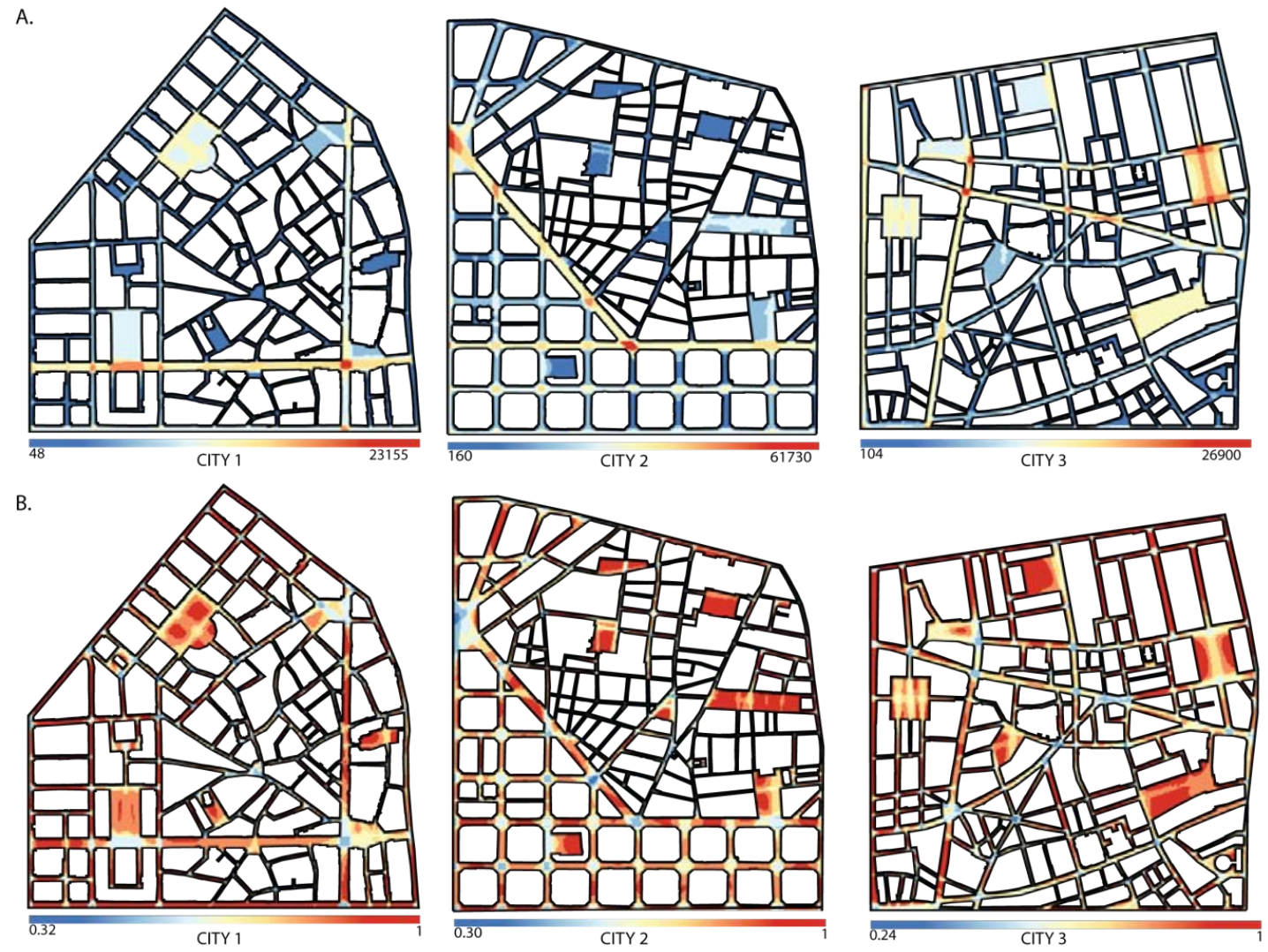

Figure 4. Visibility graph analysis (A) of the isovist area and (B) of the clustering coefficient.

\subsubsection{Behavioral Analysis}

The data analyses are focused on the moment of self-localization. The behavioral effect of interest is the button-release moment; the moment when participants spontaneously decide to change their course of action, just before their response, by releasing the button of forward movement since they believe they have oriented themselves.

To examine whether participants tend to release the button of forward movement at random or specific locations we examined the frequency distribution of the isovist values at the button-release location. In other words, to investigate where it is more likely for participants to recognize where they are, we examined whether the isovist values at the button-release location fall within the highest or lowest values of all isovist generating locations traversed by the participant during the specific trial. We 
calculated the percentiles of the population of all isovist points travelled by each participant in each trial and separated the data into 10 bins of equal number of isovist generating locations. We then normalized the isovist values at the button-release location in relation to the maximum value of all isovist points in each trial. Thus, we were able to examine in which bin there is a higher frequency of button-releases.

The behavioral analysis further examines the transitions of the perceived visuospatial information preceding the button-release moment. If any of the isovist measures of interest drive spatial orientation, then we expect that peak values within a $3 \mathrm{~s}$ time-window ${ }^{12}$ close to the button-release moment (i.e. $\left.-3 \mathrm{~s}: 0 \mathrm{~s}\right)$ to be significant different from the peak values within preceding time-windows of equal duration. We, thus, identified the peaks in the measures of interest during time-windows of $3 \mathrm{~s}$ duration for three distinct time-periods (-9s:-6s, $-6 \mathrm{~s}:-3 \mathrm{~s}$, and $-3 \mathrm{~s}: 0 \mathrm{~s})$. For each time-window we used the maximum value of change in isovist area (maximum positive peak) and the minimum value of the clustering coefficient (maximum negative peak) and then averaged separately for each participant across the time-windows of the specific timeperiod.

Because participants' speed was not controlled and varied between trials data was also assessed according to distance traveled to unpick any issues of variability in the mean peak isovist values that might be associated with speed differences. Thus, based on the mean distance traveled during a 3 s period $(M=14.92$ virtual meters, $S E=0.50)$, the same analysis was performed for three distinct distance-based segments (45m: $-30 m,-30 m:-15 m$ and $-15 m: 0 m)$. A one-way repeated measures ANOVA was

\footnotetext{
${ }^{12}$ Motivated by findings which suggest that spatial information processing related to a specific location (e.g. junction) begins up to 3 s before entering that location (Meilinger, 2008) we used a 3 s window of observation.
} 
used to compared peak isovist values between the different time-periods and between the different distance-based segments.

\subsubsection{Spatial and Brain Dynamics Analyses}

Two different approaches were adopted for the stimulus-related and response-related EEG analyses: in the first case, we used the environmental information to investigate the change in power of the EEG signal, and in the second, we used the change in EEG power to investigate the environmental correlates.

\section{Stimulus-related analyses.}

Sudden transitions of the perceived visuospatial information within a $3 \mathrm{~s}$ timewindow before the button-release moment, were used as time-locking events to examine associated differences in EEG power (stimulus-locked). In other words, we focused on the maximum positive value of change in isovist area, within a $3 \mathrm{~s}$ time-window, and maximum negative in the case of the clustering coefficient. In each trial, we are interested in the highest peak (positive and negative, respectively for the two measures) and the absolute change of the value (i.e. the difference between the values of the isovist measure at the current and the previous isovist generating location), which was used to classify the trials into two conditions of environmental change: trials where the absolute value of change falls (a) above or (b) below the median value of all trials.. The range of absolute change in isovist area was 0.73 to $270.42(M d n=439.52, n=304)$ and in clustering coefficient was $\sim 0$ to $0.42(M d n=0.047, n=307)$. We then investigated the difference in EEG power between these two conditions of environmental change. The relative changes in mean EEG power were averaged separately for each participant and condition. Epochs expanded from $-1.5 \mathrm{~s}$ to $1.5 \mathrm{~s}$ after the time-locking event. 
Motivated by the literature on movement-related and memory-relation oscillations during navigation, we focused on the delta/theta EEG power over the frontal region of the brain. The first part of the analysis was focused on the average EEG power across all four frontal/frontocentral channels (F3, F4, FC5, and FC6) and the second part on EEG power averaged across the set of left (F3 and FC5) and right (F4 and FC6) channels. Average changes in mean EEG power were compared between the two conditions of environmental change for each isovist property.

To ascertain that any difference in EEG power between the two conditions of environmental change are associated with the Aha! moment of spatial reorientation and are not driven by differences in optic flow we also included in our analysis a control condition. In other words, if any effects in EEG power between the two condition are not related to the Aha! moment and are not specific to the period preceding the buttonrelease moment then we would expect to find a similar effect during other navigation periods. Therefore, as a control condition we followed the same stimulus-related analysis but we focused on a 3 s time window immediately prior (-6s :-3s) to our test condition (-3s :0s before button-release) and examined again the EEG power differences between the two conditions of environmental change for each isovist measure. For both the test and control condition of the stimulus-related analyses, we used the Friedman's test to compare repeated-measures EEG power differences because the assumption of normality was violated (Shapiro-Wilk test $\mathrm{p}<0.05$ ).

\section{Response-related analyses}

For the response-related analysis, trials were classified into two groups, based on the changes in EEG power at the theta $(4-7 \mathrm{~Hz})$ and alpha $(8-13 \mathrm{~Hz})$ frequency bands, to examine related differences in isovist values at the button-release location. A two-way repeated-measures ANOVA with levels of channel and condition was used to analyze 
the values of the clustering coefficient at the button-release location. However, in the case of change in isovist area the assumption of normality was violated (Shapiro-Wilk test $p<0.05)$ and thus we used the Friedman's test to compare the repeated-measures.

We also looked at differences in mean trial duration and total distance traveled up to the button-release moment because participants' decision to respond to the task could be the result of the amount of visuospatial information acquired up to that point rather than the spatial local properties.

The analysis was focused: (a) on the frontocentral channels (FC5 and FC6) where the test condition includes trials that exhibited an increase in mean theta power (event-related synchronization, ERS) during the 3s time-window before the response versus trials showing a theta decrease (event-related desynchronization, ERD); and (b) on the parietal channels (P7 and P8) where the test condition includes trials that exhibited a decrease in mean alpha power (alpha ERD) during the same time-period versus the alpha ERS cases. We first compared cases in which both channels exhibited either a decrease or an increase in mean EEG power and we then focused on the recordings of each channel to examine hemispheric differences.

\subsubsection{Statistical Analysis}

Data was analyzed using the IBM SPSS software (IBM SPSS, v 25.0. Armonk, NY: IBM Corp.). Analyses of the collected data were focused on the within-subject factors because the same participants provided data points for all conditions analyzed. P-values $<.05$ were considered statistically significant. The error bars display standard errors of the mean (SE). Raincloud plots (Micah et al., 2019) illustrate data points of the averaged values for each participant, their distribution and the mean. 


\section{Results}

\subsection{Performance}

Participant's $(n=20)$ performance shows that they were able to perform well and engage with the task during the experiment. Correct responses $(M=70.85 \%, S E M=5.31 \%)$, were significantly greater, $t(19)=10.21, p<.001, d=2.28$, than the chance level of $16.67 \%$. Participants were able to encode the map of each environment and the patterns of the street networks without difficulty since they press the 'ready' button relatively quick during the study-map phase (mean duration: $57 \mathrm{~s}$, range: $15 \mathrm{~s}-229 \mathrm{~s}$ ). The SBSOD scores show no correlation with participants' percentage of correct responses, $r(18)=-.04$, $p=.87$, or mean confidence ratings, $r(18)=.27, p=.26$ across participants, and no correlation was found between percentage of correct responses, and age, $r(18)=.35$, $p=.13$. To further investigate these relationships, we also examined for possible correlations across trials. There is a positive correlation between confidence ratings across trials ${ }^{13}$ and SBSOD scores, $r(398)=.19, p<.001$ but no correlation between age and confidence ratings, $r(398)=.03, p=.63$, which might be due to the fact that participants were all relatively young (mean age: 33 years old).

In general, participants felt certainty about their position in space (confidence ratings: $M=2.30, S E=0.09$ ) with $52 \%$ of all responses rated as certain, $27 \%$ as probably right, and $21 \%$ of responses were rated as not sure. The $1 \times 3$ repeated measures ANOVA found a significant difference in the mean angular error of each participant between the different confidence rating categories, $F(2,28)=16.46, p<.001, \eta^{2}=.54$. Post-hoc pairwise comparisons using Bonferroni correction confirmed that trials with

\footnotetext{
${ }^{13}$ Participants did not give a confidence rating of their response in some trials and thus the number of trials with information on confidence ratings is 398 instead of 464 .
} 
confidence rating certain had a significantly lower angular error $(M=19.00$ degrees, $S E=4.79)$ than trials with confidence rating probably right $(M=41.21$ degrees, $S E=8.43$, adjusted $p=.047, d=-.84)$ and not sure $(M=77.26$ degrees, $S E=9.62$, adjusted $p<.001$, $d=-1.98$ ). Mean angular errors of trials with confidence rating probably right were also significantly lower than in trials with confidence rating not sure (adjusted $p=.031, d=$ $1.03)$.

Distance traveled within each route and the duration of each trial might indicate the amount of spatial information that an individual was able to acquire during each trial which in turn may be related to the confidence rating of each response. However, 1x3 repeated measures ANOVA found no significant difference in either mean distance traveled, $F(2,28)=0.34, p=.71, \eta^{2}=.03$, nor in trial duration, $F(2,28)=0.08, p=.93$, $\eta^{2}<.01$, between the three categories of confidence rating.

We have also checked for consistency of variables related to participants performance across the three environments and found no significant differences. The 1x3 repeated measures ANOVA found no significant difference in the mean angular error of each participant between the three virtual environment, $F(2,38)=1.45, p=.25$. We also examined mean confidence ratings between the three environments and although the ANOVA found a significant overall effect, $F(2,38)=3.63, p=.036$, pairwise comparison of mean confidence ratings did not reach significance (all $\mathrm{p}>.05$ ). Also, the Friedman's test found no significant differences in mean duration of studymap phase, $\chi^{2}(2)=3.11, p=.21$, between the three environments. These results support the idea that there is no significant difference between the three virtual environments and the map representations of the spatial layout of each environment.

\subsection{Frequency Distribution of Button-release Responses}

Visibility graph analysis was used to quantify the visuospatial properties of the virtual 
environments, as shown in Figure 4. This enabled the study of participants' exploratory behavior, illustrated in Figure 5A, in relation to the changes in isovist area and the clustering coefficient. The frequency distributions of the isovist values at the buttonrelease locations, as shown in Figure 5B, indicate in what sort of spaces participants tend to stop more frequently to give their response to the task.

The frequency distribution of the values at button-release location in relation to the population of all isovist generating locations traversed by each participant in each trial, as shown in Figure 5B, indicate in what sort of spaces participants tend to stop more frequently to give their response to the task. There is no clear tendency for the change in isovist area and responses do not fall within the higher values of this measure. Instead the distribution appears to be random. However, in the case of the clustering coefficient there is a clear trend towards lower values: a strong concentration of responses $(30 \%)$ fall within the lowest $20 \%$ of the clustering coefficient values. The higher count of cases appears to be in the second bin, within the $10 \%-20 \%$ of the lowest clustering coefficient values, which suggests that participants chose locations with low values of clustering coefficient but not the lowest. 
A.

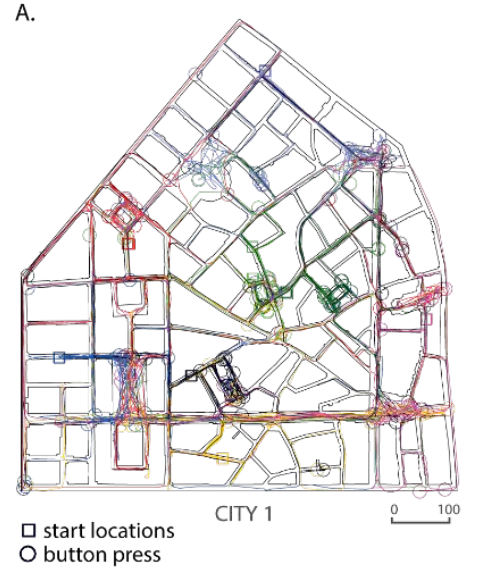

B.

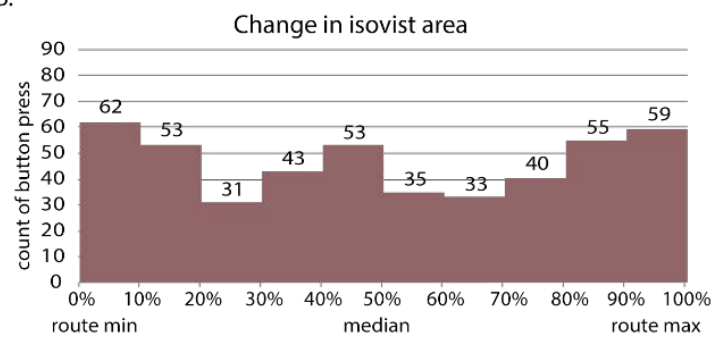

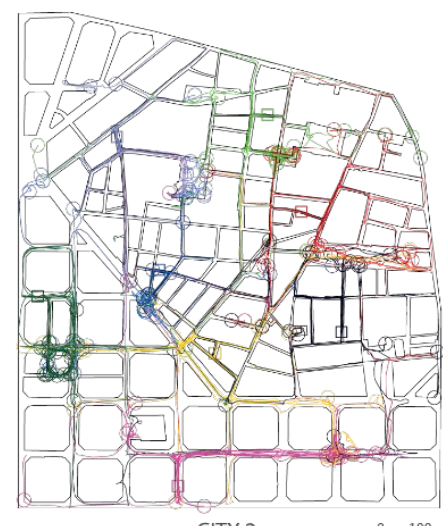

CITY 2

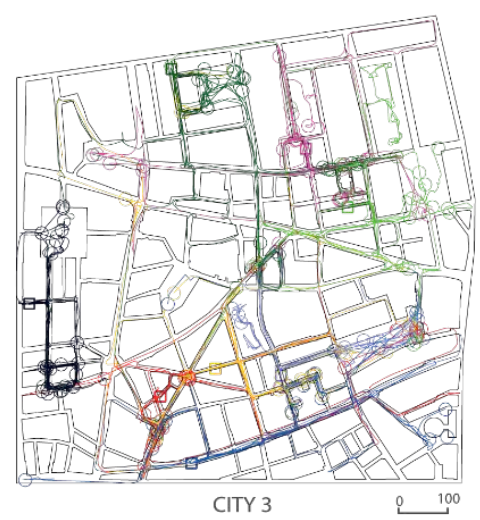

CITY 3

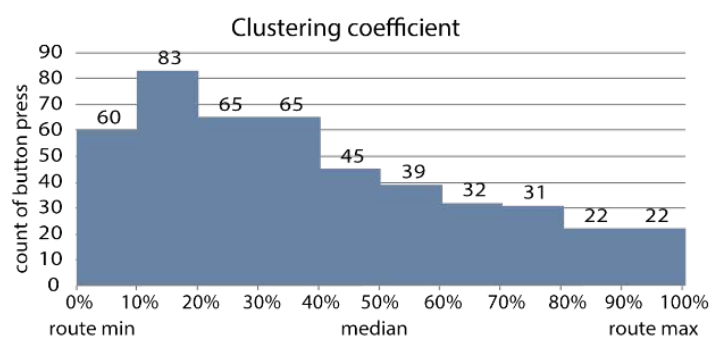

Figure 5. A. Participants' exploratory paths. Colors represent paths from the same starting location. Order of starting locations $(n=8)$ was randomized for each participant; B. Count of responses based on isovist measurements at the button-release location relative to the distribution of these values in each trial. The isovist values at the button-release location are normalized in relation to the maximum value of all isovist generating locations in each trial (x/max).

\subsection{Isovist Properties Driving Spatial Reorientation}

The maximum change in isovist area and the minimum clustering coefficient within time-windows of $3 \mathrm{~s}$ duration were compared across three different time-periods of equal duration that precede the button-release moment, as shown in Figure 6A. A one-way repeated measures ANOVA found an overall significant difference between the three time-periods for the clustering coefficient ${ }^{14}, F(1.51,28.77)=14.69, p=.005, G-G \varepsilon=$ $.75 \eta^{2}=.28$ but only a trend in the case of change in isovist area, $F(2,38)=3.12, p=.056$.

Post-hoc pairwise comparisons using Bonferroni correction confirmed that the means of

${ }^{14}$ In this case the assumption of sphericity was not met (Mauchly's test of sphericity, p-value $<0.05$ ) and, thus, we report the Greenhouse-Geisser adjusted degrees of freedom, p-values, and epsilons $(\mathrm{G}-\mathrm{G} \varepsilon)$. 
clustering coefficient within the $-3 \mathrm{~s}: 0$ s time-period $(M=0.64, S E=0.007)$ are

significantly lower compared to the $-6 \mathrm{~s}:-3 \mathrm{~s}$ time-period $(M=0.67, S E=0.009$, adjusted $p=.017, d=.67)$ and the $-9 \mathrm{~s}:-6 \mathrm{~s}$ time-period $(M=0.67, S E=0.009$, adjusted $p=.032, d=$ $.73)$.

To ascertain that any significant differences in isovist values are not the product of variations in distance traveled, we also compared mean peak values of the isovist measures across three different distance-based segments of $15 \mathrm{~m}$ length each (Figure 6B). A one-way repeated measures ANOVA found significant difference for the clustering coefficient $F(2,38)=15.32, p<.001, \eta^{2}=.53$ but no significant difference for the change in isovist value, $F(2,38)=0.51 p=.60$. Post-hoc pairwise comparisons using Bonferroni correction confirmed that mean minimum values of the clustering coefficient within the $-15 \mathrm{~m}: 0 \mathrm{~m}$ segment $(M=0.64, S E=0.007)$ are significantly lower than within the $-30 \mathrm{~m}:-15 \mathrm{~m}$ segment $(M=0.69, S E=0.008$, adjusted $p<.001, d=1.32)$ or within the $-45 \mathrm{~m}:-30 \mathrm{~m}(M=0.69, S E=0.01$, adjusted $p=.001, d=1.29)$.

Thus, the minimum clustering coefficient is significantly lower, across participants, within the window of observation closer to the button release moment lower for both time-based and distance-based behavioral analyses. The following sections examine if the related brain dynamics (stimulus-locked and response-locked) provide further evidence regarding the contribution of each isovist measure to the moment of reorientation. 

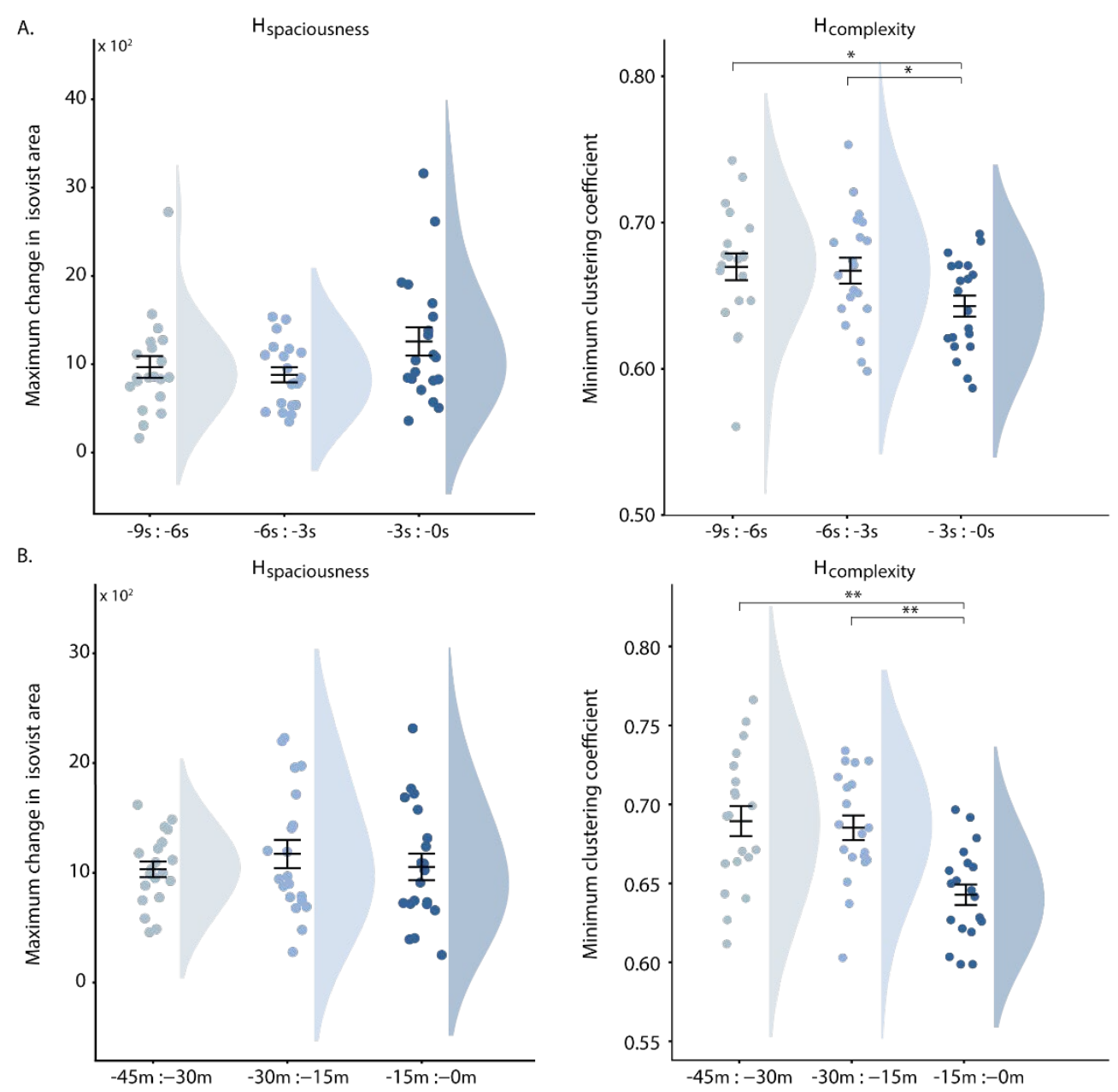

Figure 6. Raincloud plots of mean peak isovist values during (A) three time-periods of $3 \mathrm{~s}$ duration and (B)three distance segments of $15 \mathrm{~m} * \mathrm{p}<0.05, * \mathrm{p}<0.01$ )

\subsection{Differences in Brain Dynamics Associated with Peak Changes in Isovist Values}

The stimulus-related analysis examines the event-related potentials (ERPs) and the EEG power $(2-7 \mathrm{~Hz})$ at the frontal/frontocentral region between two conditions of transitions in the perceived visuospatial information (below or above median, as explained in Methods section 2.5.4). Based on visual inspection of the ERPs (Figure 7A), these differences appear to be more pronounced in the case of the clustering coefficient, especially for the channels (F4 and FC6) of the right hemisphere. 
A non-parametric Friedman test ${ }^{15}$ was used to examine the differences in the mean EEG power $(2-7 \mathrm{~Hz})$-averaged across all four frontal and frontocentral channelsbetween the two conditions and the two isovist measures, as shown in Figure 7Bi. The Friedman test found a significant difference in mean EEG power, $\chi^{2}(3)=16.62, p=.001$, and post-hoc test using a Wilcoxon signed-rank test, with a Bonferroni correction, were used to follow up this finding. There was a significant difference in mean EEG power $(z=-2.50$, adjusted $p=.025, r=-.40)$ between trials with a peak change in isovist area that falls below the median $(M d n=-0.40 \mathrm{db})$ versus above the median $(M d n=0.16 \mathrm{db})$. Trials with peak changes in the clustering coefficient that fall above the median have a significant increase $(M d n=0.20 \mathrm{db})$ in delta-theta power $(z=-2.88$, adjusted $p=.008, r=-$ .46) when compared to trials with peak changes that fall below the median $(M d n=-$ $0.32 \mathrm{db})$.

Because in the case of the clustering coefficient the ERP waveforms of the right channels appear to have significant differences for a larger time-window, we also examined the EEG power of the left (Figure 7Bii) and right (Figure 7Biii) channels separately. The Freidman's test found a significant difference in mean EEG power, between the two conditions, of left, $\chi^{2}(3)=20.94, p<.001$ and right channels, $\chi^{2}(3)=12.78, p=.005$. Post-hoc Wilcoxon signed-rank tests, with a Bonferroni correction, confirmed that average EEG power across the right channels is significantly higher for peak changes in the clustering coefficient $(z=-2.43$, adjusted $p=.02, r=-.38)$ that fall above the median $(M d n=0.16 \mathrm{db})$ compared to peak isovist changes that fall below the median, which were associated with a delta/theta decrease $(M d n=-0.26 \mathrm{db})$.

\footnotetext{
${ }^{15}$ Because the assumption of normality was violated (Shapiro-Wilk test $p<0.05$ ) we used nonparametric tests for all the comparisons reported in this section.
} 
There was no significant difference in EEG power of the right channels for the change in isovist area $(z=-2.02$, adjusted $p=.09, r=-.32)$. Left frontal/frontocentral channels exhibited a significant decrease in mean EEG power for trials with a peak change that falls below the median for both the isovist area $(z=-2.91$, adjusted $p=.007, r=-.46, M d n$ $=-0.18 \mathrm{db})$ and the clustering coefficient $(z=-3.21$, adjusted $p=.003, \mathrm{r}=-.50, M d n=-$ $0.29 \mathrm{db})$ compared to cases where peak isovist change was above the median ( $M d n=$ $0.04 \mathrm{db}, M d n=0.03 \mathrm{db}$, respectively).

For the control condition we followed the same approach. We examined the EEG power differences between the two conditions of environmental change (below vs. above median) for each isovist measure but we focused on peak changes within the time-period of $-6 \mathrm{~s}$ to $-3 \mathrm{~s}$. The Freidman's test found no significant difference in the mean EEG power $(2-7 \mathrm{~Hz})$-averaged across all four frontal and frontocentral channelsbetween the two conditions and the two isovist measures, $\chi^{2}(3)=2.64, p=.45$. Also, the Freidman's test found no significant differences between the two conditions in mean EEG power of the left, $\chi^{2}(3)=0.60, p=.90$ nor the right channels, $\chi^{2}(3)=2.55, p=.47$. 
A. Event-related potentials

- peak changes in isovist value $<$ median -.-.-.

peak changes in isovist value $>$ median
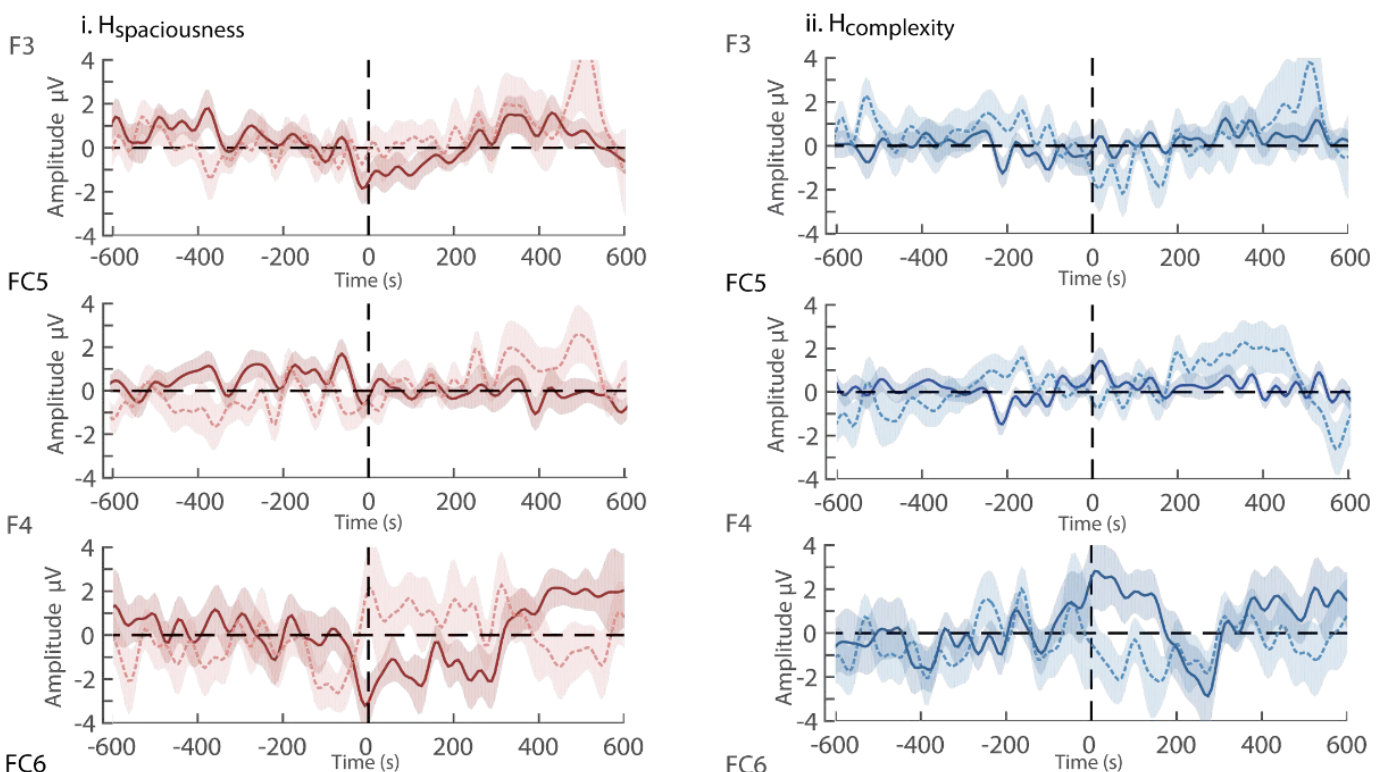

FC5

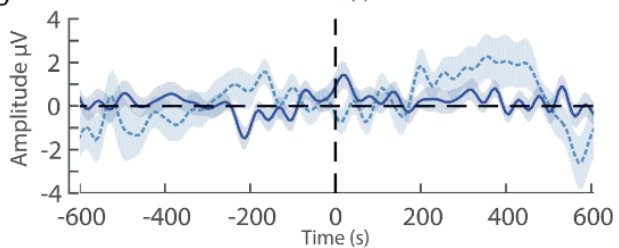

F4
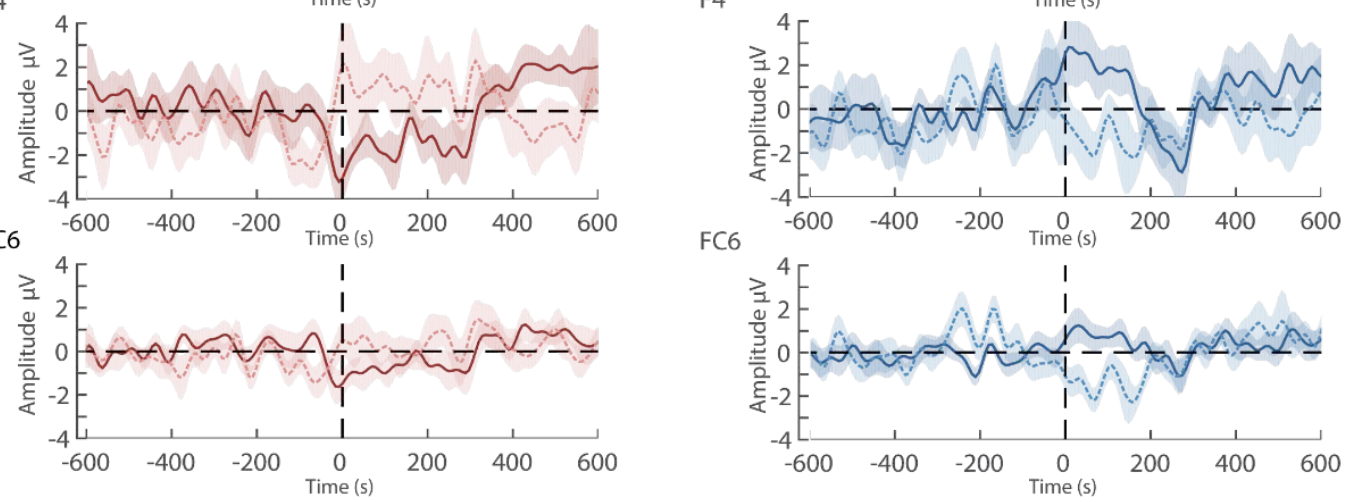

B. Mean delta/theta EEG power difference

$1=$ peak changes in isovist value $<$ median

$2=$ peak changes in isovist value $>$ median
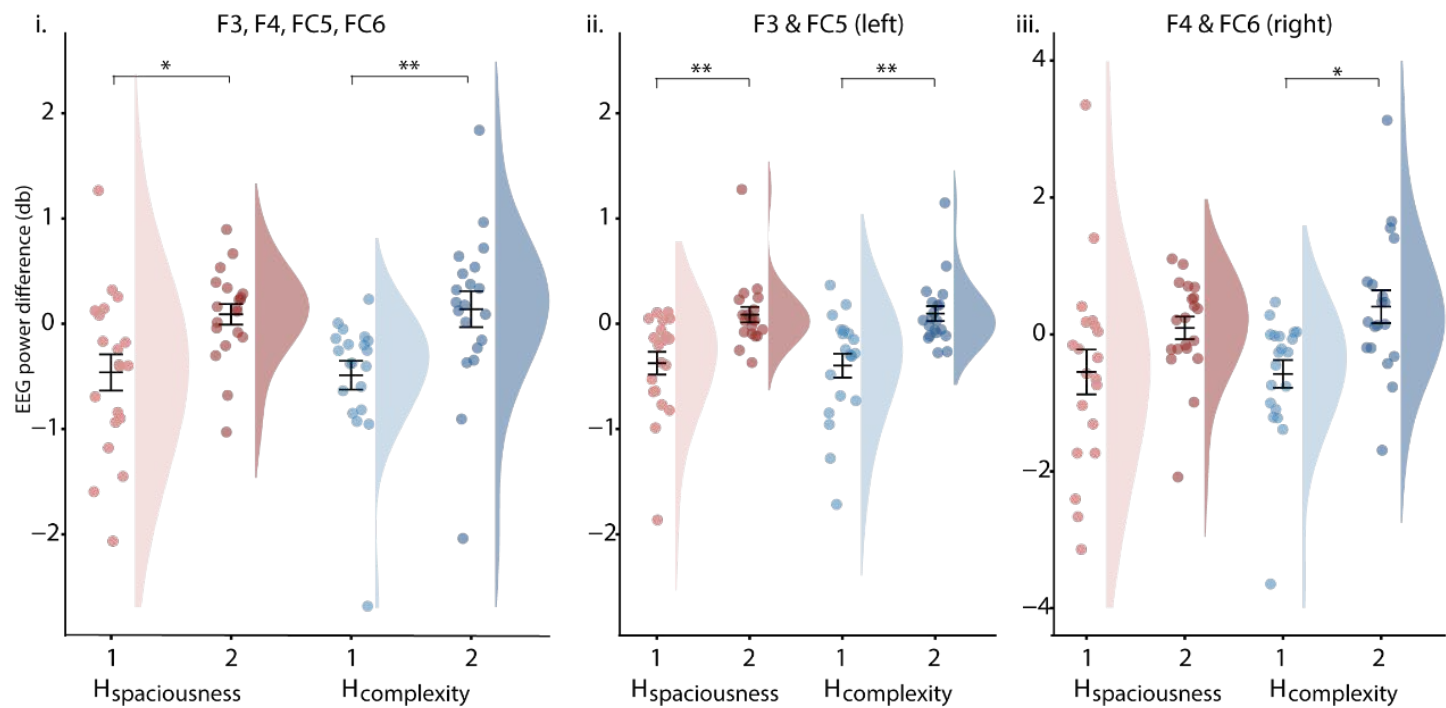

Figure 7. A. Event-related potentials for peak changes in area and the clustering coefficient that fall below the median (dotted line) and above the median (solid line); B. Raincloud plots of mean delta/theta power difference averaged over all frontal/ frontocentral channels (i), left channels (ii) and right channels (iii) for the two conditions of environmental change * $p<0.05, * * p<0.01)$. 


\subsection{Isovist Values Associated with Differences in Response-related Brain Dynamics}

A 2 × 2 repeated measures ANOVA (conditions by set of channels) was used to test for differences in isovist values at the button-release location between cases that exhibited alpha ERD versus alpha ERS in both parietal channels (P8 \& P7) and cases with theta ERS versus theta ERD in both frontocentral channels (FC5 \& FC6), as shown in Figure 8A. The analysis of variance found a significant effect of condition in the case of the clustering coefficient for the two sets of channels, $F(1,18)=11.46, p=.003, \eta^{2}=.39$. Post-hoc paired t-tests, with Bonferroni correction, confirmed that the clustering coefficient is significantly lower, $t(18)=-2.53$, adjusted $p=.042, d=-.75$, for trials with a parietal alpha decrease $(M=0.69, S E=0.02)$ compared to the parietal alpha increase condition $(M=0.74, S E=0.02)$. Similarly, the clustering coefficient is significantly lower, $t(19)=-2.60$, adjusted $p=.036, d=-.81$ for the frontocentral theta increase condition $(M=0.69, S E=0.02)$ compared to trials that exhibited a frontocentral theta decrease $(M=0.75, S E=0.02)$. The Friedman's test ${ }^{16}$ found no significant differences for the change in isovist area, $\chi^{2}(3)=1.52, p=.68$. Also, no significant differences were found in mean distance travelled, $t(19)=-0.87, p=.40, d=-.20$ nor in mean trial duration, $t(19)=-0.51, p=.61, d=-.10$ between the two conditions for either alpha power in parietal channels, nor theta power in frontocentral electrodes, $t(19)=-1.79, p=$ $.09, d=-.27$ and $t(19)=-1.65, p=.12, d=-.15$, respectively.

Additionally, trials were classified based on EEG power differences in each individual electrode, shown in Figure 8B, which enables investigating hemispheric differences. The Friedman's test found no significant differences for the change in

\footnotetext{
${ }^{16}$ Because data related to the change in isovist area was not normally distributed (Shapiro-Wilk test $p<0.05$ ) we used non-parametric tests.
} 
isovist area for either the case of parietal channels, $\chi^{2}(3)=3.78, p=.29$ nor for the case of the frontocentral channels, $\chi^{2}(3)=4.44, p=.22$. The $2 \times 2$ repeated measures ANOVA (conditions by channels) found a significant main effect on condition, in the case of the clustering coefficient, for frontocentral channels, $F(1,19)=4.47, p=.048, \eta^{2}=.19$, but post-hoc paired t-tests with Bonferroni correction did not reached significance for neither FC5, $t(19)=-2.00$, adjusted $p=.122, d=-.60$ nor FC6, $t(19)=-1.53$, adjusted $p=.284, d=-.52$. For parietal channels, there was a significant main effect of condition in the case of the clustering coefficient, $F(1,19)=6.61, p=.02, \eta^{2}=.26$. Post-hoc paired $t$ tests showed no significant difference for $\mathrm{P} 7, t(19)=-1.59$, adjusted $p=.26, d=-.50$. However, trials with alpha ERD in the right channel, P8, have significantly, $t(19)=-$ 2.47 , adjusted $p=.046, d=-.61$, lower clustering coefficient values $(M=0.69, S E=$ $0.01)$ compared to trials with an increase in alpha power $(M=0.72, S E=0.01)$. Again, no significant differences were found in mean distance travelled, $t(19)=-1.32, p=.20, d$ $=-.25$, nor in mean trial duration, $t(19)=-0.85, p=.40, d=-.11$, between the two conditions of alpha power in the right parietal channel, which suggests that these differences in response-related brain dynamics are rather a spatial boundary effect. 
A. i. Hspaciousness

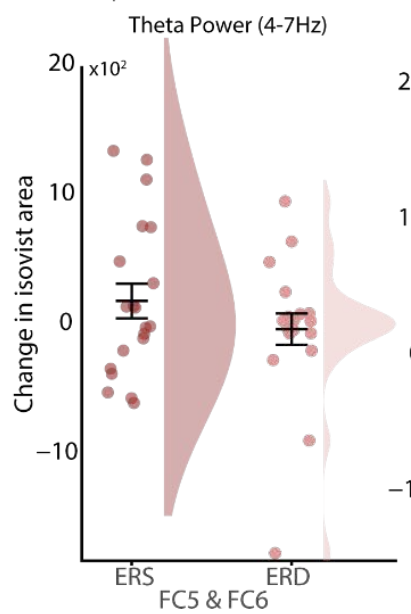

B. i. $\mathrm{H}_{\text {spaciousness }}$
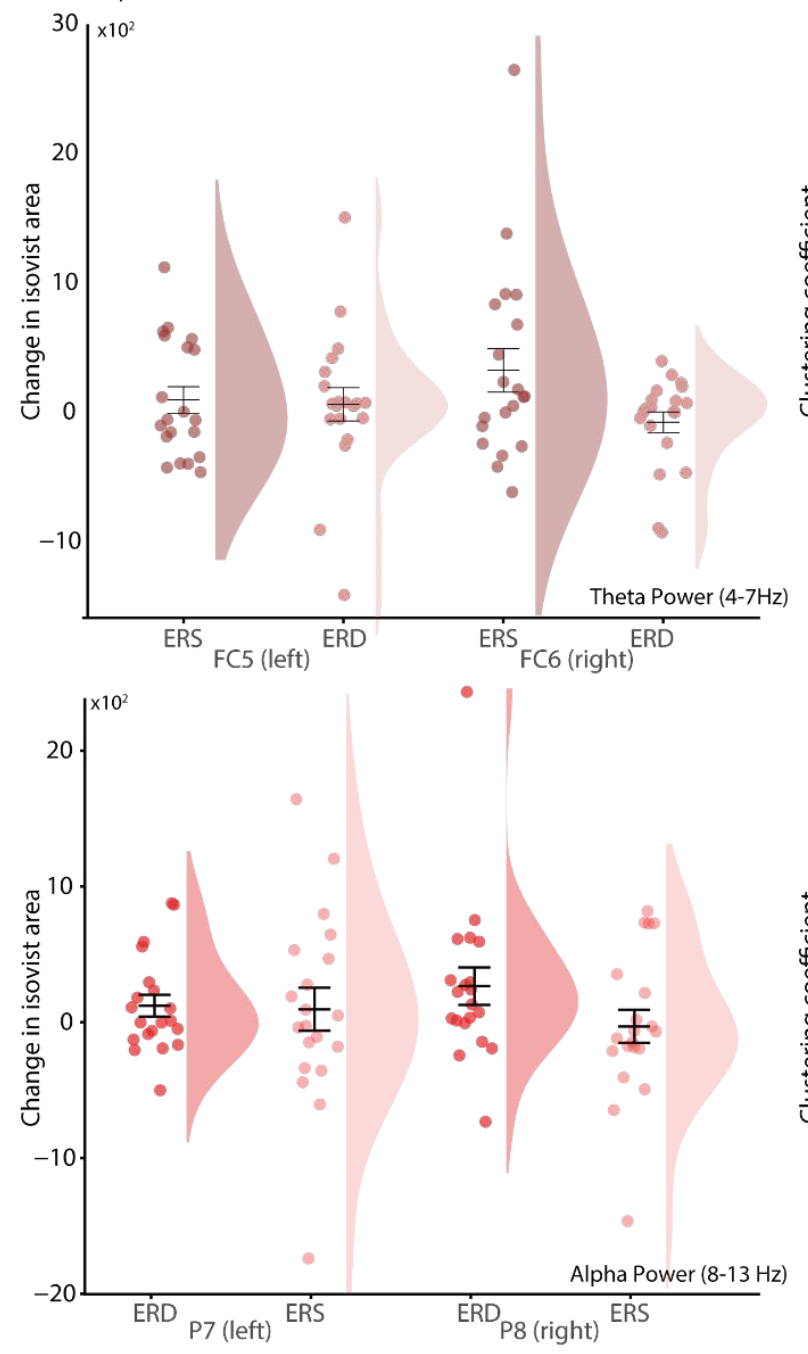

ii. Hcomplexity

Theta Power $(4-7 \mathrm{~Hz})$

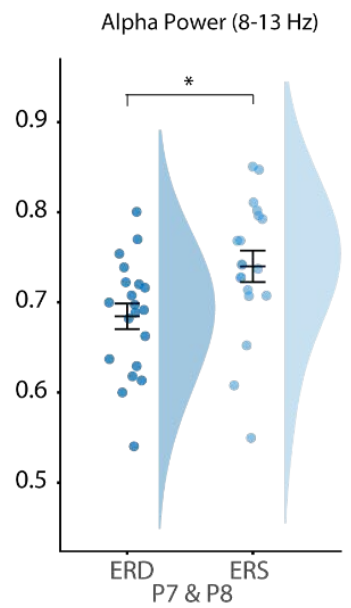

ii. $\mathrm{H}_{\text {complexity }}$
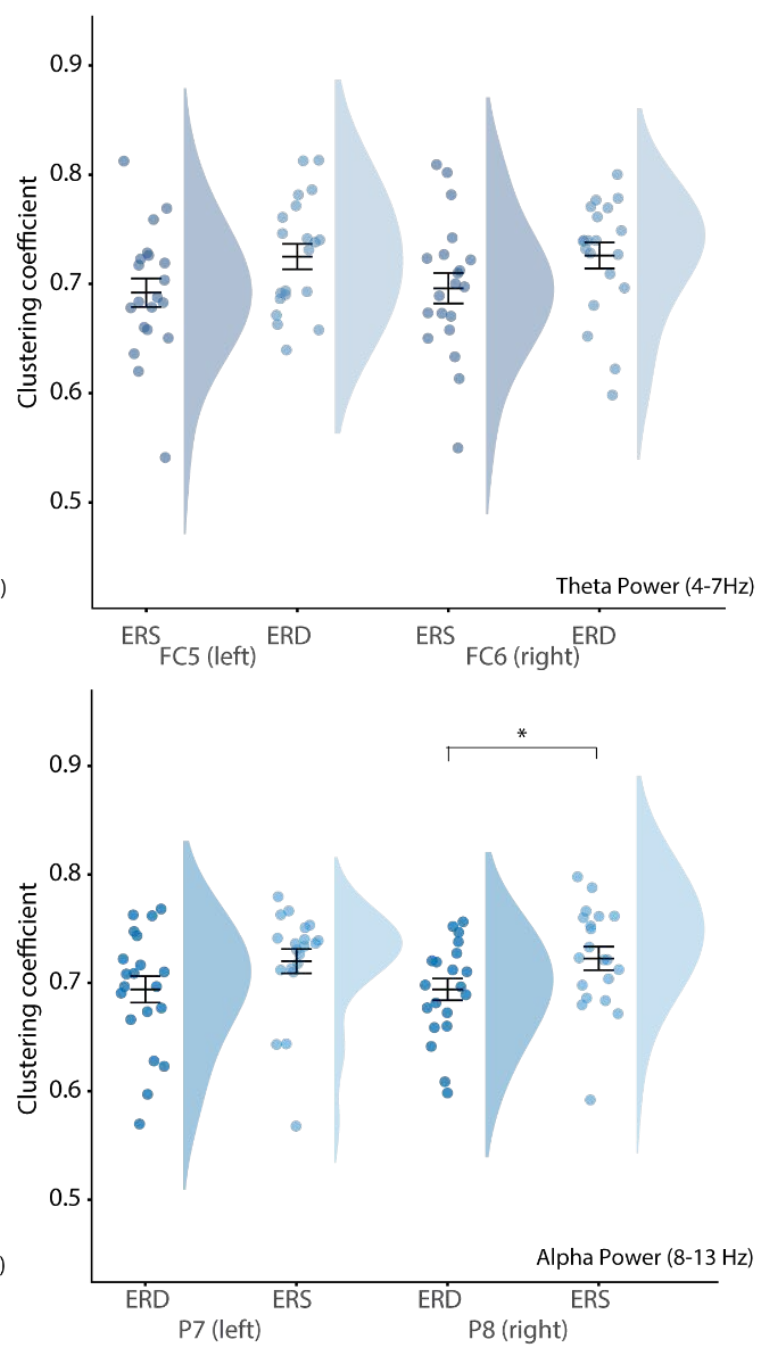

Figure 8. A. Raincloud plots of mean values of change in isovist area (left) and the clustering coefficient (right) for theta ERS versus theta ERD in both frontocentral channels (FC5 \& FC6) and alpha ERD versus alpha ERS in both parietal channels (P7 \& P8); B. Raincloud plots of mean values of (i) change in isovist area and (ii) the clustering coefficient (top) for theta ERS versus theta ERD in each frontocentral channel and (bottom) for alpha ERD versus alpha ERS in each parietal channel $(* p<0.05)$. 


\section{Discussion}

The Aha! experience of reorientation in large-scale urban environments is a relatively under-investigated phenomenon within the field of wayfinding. We developed an experimental design that was as close as possible to a real-world scenario to provide a range of possibilities that would allow the Aha! experience to emerge. In the experiment reported in this paper, participants' spatial updating was disrupted because they were suddenly teleported to different locations and thus their frame of reference was not immediately anchored to the external environment (or the map of the environment). They were able to move freely within the virtual environment, presumably looking for visuospatial cues that would facilitate their reorientation and self-localization relative to the map. Thus, they were able to respond to the task in an ethological way.

The behavioral results show that participants tend to stop to give their response just after passing through locations (within $-3 \mathrm{~s}: 0 \mathrm{~s} /-15 \mathrm{~m}: 0 \mathrm{~m}$ ) where the clustering coefficient values are significantly lower than during the preceding $-6 \mathrm{~s}$ : $-3 \mathrm{~s}$ time-period/ -30m: -15m distance-segment. In contrast, there was no significant difference for the change in isovist area. Peak changes that fall above the median value for both isovist measures during the $-3 \mathrm{~s}$ : $0 \mathrm{~s}$ time-window are associated with an increase in delta-theta power over frontal/frontocentral areas. In addition, there is a significant increase in delta/theta in the channels of the right hemisphere but only when there is a large drop in the clustering coefficient values. Therefore, these results suggest that both measures appear to contribute to the moment of reorientation; but their contribution may be different.

One alternative explanation is that the effects between the two conditions of environmental change might be driven by differences of the patterns of eye movement artifacts, which are likely to be different between the two conditions. Although we used 
ICA decomposition to remove eye artifacts during preprossesing, we cannot exclude fully the possibility. However, if the effects were mainly driven by differences in saccadic behavior between conditions then we would expect similar results for the control condition. Another possibility is that these differences in EEG power are associated only with the transitions in the perceived environmental features and are independent of the Aha! moment of reorientation. In this case again one would expect such correlations between the delta/theta EEG power and the isovist measures during any other navigation period, such during the -6 s to -3 s time-period. However, there are no such differences in EEG power between the two cases of environmental change for the control condition.

The analysis of the relationship between response-related brain dynamics and the isovist values at the button-release location shows that the clustering coefficient has a stronger link to the Aha! moment of reorientation than the change in isovist area. The frequency distribution of isovist values at the button-release location shows that most responses occur at the location where the clustering coefficient values are within the lowest percentiles of the whole environment, but there is no clear trend for the changes in isovist area. Increases in frontocentral theta activity, assumed to be related to conflict resolution processes, and parietal alpha decrease, likely reflecting translation of spatial information between reference frames, are associated with significantly lower values of the clustering coefficient at the button-release location. However, there is no significant difference in the case of change in isovist area.

An alternative hypothesis that could be raised is that both spatial conditions - a change in spaciousness and a change in complexity - may contribute in different ways to spatial reorientation. For example, when approaching a typical street junction there is a sequence of events taking place: first, a sudden increase in area when entering the 
junction, and then, after a certain time interval, once one is at the center of the junction a location with low clustering coefficient. The investigation of a possible sequential dependency between these two conditions; a change in spaciousness followed by a change in complexity, might be an interesting direction for future research.

Local properties of the spatial configuration appear to have a significant contribution to the emergence of the spatial Aha! moment. Nevertheless, it is important to note here that other factors may influence the occurrence of this event such as the socio-cultural and geographical environment and other individual differences, such as spatial abilities and age (Gramann, 2013; Heft, 2013).

In general, the results suggest that the Aha! experience of reorientation is a spatial boundary effect. Differences in the response-related brain dynamics are associated with differences in the local visuospatial conditions but not the amount of visuospatial information acquired up to the moment of response. This is not surprising since the Aha! moment is considered as incidental one-shot learning that requires a representational switch, as opposed to incremental associative learning processes (Ash et al., 2012; Luo et al., 2006). Similarly, spatial learning in relation to environmental boundaries, which is supported by the hippocampus, does not follow the rules of associative learning (as landmark-based learning) but is also considered to be incidental (Burgess, 2008; Doeller \& Burgess, 2008). Environmental boundaries play a key role in spatial learning and orientation (Montello, 2007) and the clustering coefficient describes quantitatively the 'spikiness' of the environmental shape. Accurate knowledge of place location and processing of place-related spatial information are associated with activity in the right hemisphere (Igloi et al., 2010; Maguire et al., 1998), which might explain why there is a stronger association between differences in the clustering coefficient 
values and changes in EEG power in the right hemisphere channels (i.e. alpha ERD at P8, delta-theta ERS at F4 \& FC6).

Consequently, locations with low clustering coefficient values seem to act as environmental cues facilitating conflict resolution processes and the translation of spatial information between reference frames. The multidirectional fields of view at these locations appear to favor context/place recognition and spontaneous selflocalization. A conclusion that might be of practical use here is regarding the design of underground entrances. Disorientation or misorientation due to passive underground movement may persist if, for example, the geography of the underground hallway system of several exits is complex and its signage confusing. But if the underground exit is at a location that offers multidirectional fields of view then this is likely to trigger the spatial Aha! moment.

The visuospatial conditions that engender this phenomenon and the way we explore relatively unfamiliar, complex urban environments may share similarities with the way we navigate conceptual space. A growing number of studies point towards the idea that the hippocampal map may not be just for spatial memory but a 'memory space' or a 'memory map' (Buzsáki \& Moser, 2013; Eichenbaum et al., 1999; Jeffery, 2018). Recent work suggests that the hippocampus supports map-like knowledge structures organized not only in space and time but conceptual dimensions as well (Aronov et al., 2017; Bellmund et al., 2018; Buzsáki \& Moser, 2013; Constantinescu et al., 2016; Eichenbaum \& Cohen, 2014; Garvert et al., 2017) and may encode expectations regarding future experiences (Schacter et al., 2007; Stachenfeld et al., 2017).

Empirical studies on creative insights have consistently reported temporal lobe activity, reflecting the recognition of available novel associations, and the involvement of the hippocampus and parahippocampal cortex (Milivojevic et al., 2015; Salvi et al., 
2020; Shen et al., 2017; Zhao et al., 2013). Also, increased activity in the anterior cingulate cortex reported during creative insights (Mai et al., 2004; Qiu et al., 2006; Shen et al., 2018; Zhao et al., 2013), reflecting conflict resolution process (e.g. of misaligned representations) and during wayfinding before spontaneous 'backtracking' behavior (Javadi et al., 2019), often observed during reorientation. The brain's navigational system thus is likely to be involved not only in the representation of physical space but of social and conceptual space as well, supporting context retrieval and reorientation in abstract space (Epstein et al., 2017). These theories and empirical findings motivate the idea that spatial reorientation can be seen as an instance of the Aha! phenomenon.

The present study aims to show that a change in participants' behavior and brain dynamics is coupled with a change in the environmental context. A change in the amount of visual information does not appear to be sufficient to provoke the Aha! experience, even if it might trigger an element of surprise or contribute to other relevant sub-processes. In contrast, a change in the complexity of the visual information has a stronger contribution to the sudden 'bisociation' of distinct reference frames, resulting in a third hinge-dimension of experience, the Aha! moment of reorientation.

\section{Acknowledgments}

We appreciate the advice we received from Prof. Hugo Spiers (Institute of Behavioral Neuroscience, UCL) and Dr. Daniel Bush (Institute of Cognitive Neuroscience, UCL) during the initial stages of the research. We also thank Prof. Kate Jeffery (Institute of Behavioral Neuroscience, UCL) and Prof. Ruth Dalton (Lancaster University) for their feedback on the study during the viva, Dr. Daniel Bush and Dr. Jens Kandt (Centre of Advanced Spatial Analysis, UCL) for their useful feedback and comments on earlier versions of the manuscript and, of course, the people who volunteer to participate in the study. Part of this work was supported by grants from the State Scholarships Foundation (IKY) and the A.G. Leventis Foundation to the first author. The funders had no role in the conceptualization, design, data collection, analysis, decision to publish or preparation of the manuscript. 
References

Alexander, A. S., \& Nitz, D. A. (2015). Retrosplenial cortex maps the conjunction of internal and external spaces. Nature Neuroscience, 18(8). https://doi.org/10.1038/nn.4058

Araújo, D. B. d., Baffa, O., \& Wakai, R. T. (2002). Theta Oscillations and Human Navigation: A Magnetoencephalography Study. Journal of Cognitive Neuroscience, 14(1), 70-78. https://doi.org/10.1162/089892902317205339

Aronov, D., Nevers, R., \& Tank, D. W. (2017). Mapping of a non-spatial dimension by the hippocampal-entorhinal circuit. Nature, 543(7647), 719. https://doi.org/10.1038/nature21692

Ash, I. K., Jee, B. D., \& Wiley, J. (2012). Investigating Insight as Sudden Learning. The Journal of Problem Solving, 4(2), 2.

Barry, C., \& Burgess, N. (2014). Neural mechanisms of self-location. Curr Biol, 24(8), R330-339. https://doi.org/10.1016/j.cub.2014.02.049

Barton, K. R., Valtchanov, D., \& Ellard, C. (2014). Seeing Beyond Your Visual Field: The Influence of Spatial Topology and Visual Field on Navigation Performance. Environment and Behavior, 46(4), 507-529. https://doi.org/10.1177/0013916512466094

Bellmund, J., Gardenfors, P., Moser, E. I., \& Doeller, C. (2018). Navigating cognition: Spatial codes for human thinking. Science, 362(6415). https://doi.org/10.1126/science.aat6766

Bellmund, J. L. S., de Cothi, W., Ruiter, T. A., Nau, M., Barry, C., \& Doeller, C. F. (2019). Deforming the metric of cognitive maps distorts memory. Nature human behaviour. https://doi.org/10.1038/s41562-019-0767-3

Benedikt, M. L. (1979). To Take Hold of Space: Isovists and Isovist Fields. Environment and Planning B: Planning and Design, 6(1), 47-65.

Benedikt, M. L., \& Burnham, C. A. (1985). Perceiving architectural space: From optic arrays to isovists. In W. H. Warren, R. Shaw, \& E. (Eds.), Persistence and change (pp. 103-114). Hillsdale, NJ:Lawrence Erlbaum.

Berlyne, D. E. (1950). Novelty and curiosity as determinants of exploratory behaviour. British Journal of Psychology. General Section, 41(1-2), 68-80. https://doi.org/10.1111/j.2044-8295.1950.tb00262.x

Berlyne, D. E. (1960). Conflict, arousal and curiosity. New York : McGraw-Hill.

Berlyne, D. E. (1966). Curiosity and exploration. Science (New York, N.Y.), 153(3731), 25. https://doi.org/10.1126/science.153.3731.25

Berlyne, D. E. (1970). Novelty, complexity, and hedonic value. Perception \& Psychophysics, 8(5), 279-286. https://doi.org/10.3758/BF03212593

Binet, M. A. (1894). Reverse illusions of orientation. Psychological Review, 1(4), 337350. https://doi.org/10.1037/h0067333

Bischof, W. F., \& Boulanger, P. (2003). Spatial navigation in virtual reality environments: an EEG analysis. Cyberpsychol Behav, 6(5), 487-495. https://doi.org/10.1089/109493103769710514

Bohbot, V. D., Copara, M. S., Gotman, J., \& Ekstrom, A. D. (2017). Low-frequency theta oscillations in the human hippocampus during real-world and virtual navigation. Nature communications, 8, 14415-14415.

https://doi.org/10.1038/ncomms14415 
Burgess, N. (2006). Spatial memory: how egocentric and allocentric combine. Trends in Cognitive Sciences, 10(12), 551-557. https://doi.org/10.1016/j.tics.2006.10.005

Burgess, N. (2008). Spatial cognition and the brain. Ann N Y Acad Sci, 1124, 77-97. https://doi.org/10.1196/annals.1440.002

Burgess, N., Becker, S., King, J. A., \& O'Keefe, J. (2001). Memory for events and their spatial context: models and experiments. Philos Trans R Soc Lond B Biol Sci, 356(1413), 1493-1503. https://doi.org/10.1098/rstb.2001.0948

Bush, D., Bisby, J. A., Bird, C. M., Gollwitzer, S., Rodionov, R., Diehl, B., McEvoy, A. W., Walker, M. C., \& Burgess, N. (2017). Human hippocampal theta power indicates movement onset and distance travelled. Proc Natl Acad Sci U S A, 114(46), 12297-12302. https://doi.org/10.1073/pnas.1708716114

Buzsáki, G., \& Moser, E. I. (2013). Memory, navigation and theta rhythm in the hippocampal-entorhinal system. Nature Neuroscience, 16(2), 130. https://doi.org/10.1038/nn.3304

Byrne, P., Becker, S., \& Burgess, N. (2007). Remembering the Past and Imagining the Future: A Neural Model of Spatial Memory and Imagery. Psychological Review, 114(2), 340-375.

Chadwick, M. J., \& Spiers, H. J. (2014). A local anchor for the brain's compass. Nature Neuroscience, 17(11), 1436. https://doi.org/10.1038/nn.3841

Cheng, K. (1986). A purely geometric module in the rat's spatial representation. Cognition, 23(2), 149-178. https://doi.org/10.1016/0010-0277(86)90041-7

Cheng, K., Huttenlocher, J., \& Newcombe, N. (2013). 25 years of research on the use of geometry in spatial reorientation: a current theoretical perspective. Psychon Bull Rev, 20(6), 1033-1054. https://doi.org/10.3758/s13423-013-0416-1

Cheng, K., \& Newcombe, N. (2005). Is there a geometric module for spatial orientation? squaring theory and evidence. Psychonomic Bulletin \& Review, 12(1), 1-23. https://doi.org/10.3758/BF03196346

Chiu, T. C., Gramann, K., Ko, L. W., Duann, J. R., Jung, T. P., \& Lin, C. T. (2012). Alpha modulation in parietal and retrosplenial cortex correlates with navigation performance. Psychophysiology, 49(1), 43-55. https://doi.org/10.1111/j.1469$\underline{8986.2011 .01270 . \mathrm{x}}$

Cohen, M. X. (2014). Analyzing neural time series data: theory and practice [book]. Cambridge, Mass. ; London : MIT, 2014. https://doi.org/urn:isbn:9780262019873

Conroy Dalton, R., Hölscher, C., \& Turner, A. (2012). Understanding space: the nascent synthesis of cognition and the syntax of spatial morphologies. Environment and Planning B: Planning and Design, 39(1), 7-11.

Conroy, R. A. (2001). Spatial navigation in immersive virtual environments [Thesis (PhD) University of London 2001, http://www.ucl.ac.uk/library/

Constantinescu, A. O., O'Reilly, J. X., \& Behrens, T. E. J. (2016). Organizing conceptual knowledge in humans with a gridlike code. Science (New York, N.Y.), 352(6292), 1464. https://doi.org/10.1126/science.aaf0941

Dara-Abrams, D. (2006). Architecture of mind and world: how urban form influences spatial cognition. Space Syntax and Spatial Cognition - Proceedings of the Workshop held in Bremen, 24th September 2006,

Delorme, A., \& Makeig, S. (2004). EEGLAB: an open source toolbox for analysis of single-trial EEG dynamics including independent component analysis. Journal of Neuroscience Methods, 134(1), 9-21. https://doi.org/10.1016/j.jneumeth.2003.10.009 
Doeller, C. F., \& Burgess, N. (2008). Distinct error-correcting and incidental learning of location relative to landmarks and boundaries. P NATL ACAD SCI USA , 105 (15) 5909 - 5914. (2008).

Donchin, E. (1981). Surprise!... Surprise? Psychophysiology, 18(5), 493-513. https://doi.org/10.1111/j.1469-8986.1981.tb01815.x

Dudchenko, P. A. (2010). Why People Get Lost. Oxford : Oxford University Press

Duval, A. (2019). The representation selection problem: Why we should favor the geometric-module framework of spatial reorientation over the view-matching framework. Cognition, 192. https://doi.org/10.1016/j.cognition.2019.05.022

Eichenbaum, H., \& Cohen, N. J. (2014). Can we reconcile the declarative memory and spatial navigation views on hippocampal function? Neuron, 83(4), 764-770. https://doi.org/10.1016/j.neuron.2014.07.032

Eichenbaum, H., Dudchenko, P., Wood, E., Shapiro, M., \& Tanila, H. (1999). The hippocampus, memory, and place cells: is it spatial memory or a memory space? Neuron, 23(2), 209-226.

Ekstrom, A. D., \& Watrous, A. J. (2014). Multifaceted roles for low-frequency oscillations in bottom-up and top-down processing during navigation and memory. NeuroImage (Orlando, Fla.), 85(2), 667-677. https://doi.org/10.1016/j.neuroimage.2013.06.049

Emo, B. (2014). Seeing the Axial Line: Evidence from Wayfinding Experiments. Behavioral Sciences, 4(3), 167-180. https://doi.org/10.3390/bs4030167

Epstein, R. A., Patai, E. Z., Julian, J., B., \& Spiers, H. J. (2017). The cognitive map in humans: spatial navigation and beyond. Nature Neuroscience, 20(11), 1504. https://doi.org/10.1038/nn.4656

Fernández Velasco, P., \& Casati, R. (2020a). Subjective disorientation as a metacognitive feeling. Spatial Cognition \& Computation, 1-25. https://doi.org/10.1080/13875868.2020.1768395

Fernández Velasco, P., \& Casati, R. (2020b). The many faces of disorientation: a response to Daniel R. Montello. Spatial Cognition \& Computation, 1-14. https://doi.org/10.1080/13875868.2020.1772267

Franz, G., \& Wiener, J. M. (2005). Exploring isovist-based correlates of spatial behavior and experience. Proceedings of the 5th International Space Syntax Symposium Delft, NL. TU Delft Press. accepted.

Franz, G., \& Wiener, J. M. (2008). From Space Syntax to Space Semantics: A Behaviorally and Perceptually Oriented Methodology for the Efficient Description of the Geometry and Topology of Environments. Environment and Planning B: Planning and Design, 35(4), 574-592. https://doi.org/10.1068/b33050

Fyhn, M., Hafting, T., Treves, A., Moser, M.-B., \& Moser, E. I. (2007). Hippocampal remapping and grid realignment in entorhinal cortex. Nature, 446(7132), 190. https://doi.org/10.1038/nature05601

Gallistel, C. R. (1990). The organization of learning / C. R. Gallistel. Cambridge, Mass : MIT Press.

Garvert, M. M., Dolan, R. J., \& Behrens, T. E. (2017). A map of abstract relational knowledge in the human hippocampal-entorhinal cortex. eLife , 6 , Article e17086. (2017).

Gramann, K. (2013). Embodiment of Spatial Reference Frames and Individual Differences in Reference Frame Proclivity. Spatial Cognition \& Computation, 13(1), 1-25. https://doi.org/10.1080/13875868.2011.589038 
Haider, H., \& Rose, M. (2007). How to investigate insight: A proposal. Methods, 42(1), 49-57. https://doi.org/10.1016/j.ymeth.2006.12.004

Harrison, L. M., Duggins, A., \& Friston, K. J. (2006). Encoding uncertainty in the hippocampus. Neural Networks, 19(5), 535-546. https://doi.org/10.1016/j.neunet.2005.11.002

Hartley, T., Trinkler, I., \& Burgess, N. (2004). Geometric determinants of human spatial memory. Cognition, 94(1), 39-75. https://doi.org/10.1016/j.cognition.2003.12.001

Heft, H. (2013). Environment, cognition, and culture: Reconsidering the cognitive map. Journal of Environmental Psychology, 33, 14-25. https://doi.org/10.1016/j.jenvp.2012.09.002

Hegarty, M., Richardson, A. E., Montello, D. R., Lovelace, K., \& Subbiah, I. (2002). Development of a self-report measure of environmental spatial ability. Intelligence, 30(5), 425-447. https://doi.org/10.1016/S0160-2896(02)00116-2

Hermer, L., \& Spelke, E. (1994). A geometric process for spatial reorientation in young children. Nature, 370(6484), 57. https://doi.org/10.1038/370057a0

Hermer, L., \& Spelke, E. (1996). Modularity and development: the case of spatial reorientation. Cognition, 61(3), 195-232. https://doi.org/10.1016/S0010$\underline{0277(96) 00714-7}$

Hillier, B. (1998). Space is the Machine: A Configurational Theory of Architecture. Cambridge University Press.

Hillier, B., \& Hanson, J. (1984). The social logic of space. Cambridge : Cambridge University Press.

Hupbach, A., Hardt, O., Nadel, L., \& Bohbot, V. D. (2007). Spatial Reorientation: Effects of Verbal and Spatial Shadowing. Spatial Cognition \& Computation, 7(2), 213-226. https://doi.org/10.1080/13875860701418206

Hölscher, C., BroĞsamle, M., \& Vrachliotis, G. (2012). Challenges in multilevel wayfinding: a case study with the space syntax technique. Environment and Planning B: Planning and Design, 39, 63-82.

Igloi, K., Doeller, C. F., Berthoz, A., Rondi-Reig, L., \& Burgess, N. (2010). Lateralized human hippocampal activity predicts navigation based on sequence or place memory. Proc Natl Acad Sci U S A, 107(32), 14466-14471. https://doi.org/10.1073/pnas.1004243107

Jacobs, J. (2014). Hippocampal theta oscillations are slower in humans than in rodents: implications for models of spatial navigation and memory. Philosophical transactions of the Royal Society of London. Series B, Biological sciences, 369(1635), 20130304. https://doi.org/10.1098/rstb.2013.0304

Jaiswal, N., Ray, W., \& Slobounov, S. (2010). Encoding of visual-spatial information in working memory requires more cerebral efforts than retrieval: Evidence from an EEG and virtual reality study. Brain Res, 1347, 80-89. https://doi.org/10.1016/j.brainres.2010.05.086

Javadi, A.-H., Patai, E. Z., Marin-Garcia, E., Margois, A., Tan, H.-R. M., Kumaran, D., Nardini, M., Penny, W., Duzel, E., Dayan, P., \& Spiers, H. J. (2019).

Backtracking during navigation is correlated with enhanced anterior cingulate activity and suppression of alpha oscillations and the 'default-mode' network. Proceedings. Biological sciences, 286(1908), 20191016. https://doi.org/10.1098/rspb.2019.1016

Javadi, A. H., Emo, B., Howard, L. R., Zisch, F. E., Yu, Y., Knight, R., Pinelo Silva, J., \& Spiers, H. J. (2017). Hippocampal and prefrontal processing of network 
topology to simulate the future. Nat Commun, 8, 14652.

https://doi.org/10.1038/ncomms14652

Jeffery, K. J. (2007). Self-localization and the entorhinal-hippocampal system. Current Opinion in Neurobiology, 17(6), 684-691.

https://doi.org/10.1016/j.conb.2007.11.008

Jeffery, K. J. (2010). Theoretical accounts of spatial learning: A neurobiological view (commentary on Pearce, 2009). The Quarterly Journal of Experimental Psychology, 63(9), 1683-1699. https://doi.org/10.1080/17470210903540771

Jeffery, K. J. (2011). Place Cells, Grid Cells, Attractors, and Remapping. Neural Plasticity, 2011(2011). https://doi.org/10.1155/2011/182602

Jeffery, K. J. (2018). The Hippocampus: From Memory, to Map, to Memory Map. Trends in Neurosciences, 41(2), pp. 64-66.

Jepma, M., Verdonschot, R. G., van Steenbergen, H., Rombouts, S. A. R. B., \& Nieuwenhuis, S. (2012). Neural mechanisms underlying the induction and relief of perceptual curiosity. Frontiers in behavioral neuroscience, 6(2012), 5. https://doi.org/10.3389/fnbeh.2012.00005

Julian, J. B., Keinath, A. T., Marchette, S. A., \& Epstein, R. A. (2018). The Neurocognitive Basis of Spatial Reorientation. Curr Biol, 28(17), R1059-r1073. https://doi.org/10.1016/j.cub.2018.04.057

Julian, J. B., Keinath, A. T., Muzzio, I. A., \& Epstein, R. A. (2015). Place recognition and heading retrieval are mediated by dissociable cognitive systems in mice. Proc Natl Acad Sci US A, 112(20), 6503-6508. https://doi.org/10.1073/pnas.1424194112

Kaplan, R., Bush, D., Bisby, J. A., Horner, A. J., Meyer, S. S., \& Burgess, N. (2017). Medial Prefrontal-Medial Temporal Theta Phase Coupling in Dynamic Spatial Imagery. Journal of Cognitive Neuroscience, 29 (3) pp. 507-519. (2017).

Kaplan, R., Doeller, C. F., Barnes, G. R., Litvak, V., Düzel, E., Bandettini, P. A., \& Burgess, N. (2012). Movement-related theta rhythm in humans: coordinating self-directed hippocampal learning. PLoS Biol, 10(2), e1001267. https://doi.org/10.1371/journal.pbio.1001267

Kaplan, R., Horner, A. J., Bandettini, P. A., Doeller, C. F., \& Burgess, N. (2014). Human hippocampal processing of environmental novelty during spatial navigation. Hippocampus, 24(7), 740-750. https://doi.org/10.1002/hipo.22264

Kaplan, R., \& Kaplan, S. (1989). The experience of nature: A psychological perspective. Cambridge University Press.

Kaplan, S. (1987). Aesthetics, Affect, and Cognition: "Environmental Preference from an Evolutionary Perspective". Environment and Behavior, 19(1), 3. https://doi.org/10.1177/0013916587191001

Kaplan, S. (1992). Environmental preference in a knowledge-seeking, knowledge-using organism. In J. H. Barkow, L. Cosmides, \& J. Tooby (Eds.), The Adapted Mind: Evolutionary Psychology and the Generation of Culture (pp. 581-600). Oxford University Press.

Keinath, A. T., Julian, J. B., Epstein, R. A., \& Muzzio, I. A. (2017). Environmental Geometry Aligns the Hippocampal Map during Spatial Reorientation. Current Biology, 27(3), 309-317. https://doi.org/10.1016/j.cub.2016.11.046

Knoblich, G., Ohlsson, S., Haider, H., \& Rhenius, D. (1999). Constraint Relaxation and Chunk Decomposition in Insight Problem Solving. Journal of Experimental Psychology: Learning, Memory, and Cognition, 25(6), 1534-1555. https://doi.org/10.1037/0278-7393.25.6.1534

Koestler, A. (1964). The Act of Creation. Hutchinson. 
Kubat, A., Özbil, A., Özer, Ö., \& Ekinoglu, H. (2012). The effect of built space on wayfinding in urban environments: a study of the historical peninsula in Istanbul 8th International Space Syntax Symposium,, Santiago de Chile.

Kumaran, D., \& Maguire, E. A. (2006). An unexpected sequence of events: mismatch detection in the human hippocampus. PLoS Biology, 4 (12), Article e 424. (2006).

Köhler, S., Danckert, S., Gati, J. S., \& Menon, R. S. (2005). Novelty responses to relational and non-relational information in the hippocampus and the parahippocampal region: A comparison based on event-related fMRI. Hippocampus, 15(6), 763-774. https://doi.org/10.1002/hipo.20098

Lee, S., \& Spelke, E. (2010). Two systems of spatial representation underlying navigation. Exp Brain Res, 206(2), 179-188. https://doi.org/10.1007/s00221$\underline{010-2349-5}$

Lee, S., \& Spelke, E. (2011). Young children reorient by computing layout geometry, not by matching images of the environment. Psychon Bull Rev, 18(1), 192-198. https://doi.org/10.3758/s13423-010-0035-z

Lee, S. A., Sovrano, V. A., \& Spelke, E. S. (2012). Navigation as a Source of Geometric Knowledge: Young Children's Use of Length, Angle, Distance, and Direction in a Reorientation Task. Cognition, 123(1). https://doi.org/10.1016/j.cognition.2011.12.015

Lee, S. A., \& Spelke, E. S. (2010). A Modular Geometric Mechanism for Reorientation in Children.

http://www.wjh.harvard.edu/ lds/pdfs/Lee\%20and\%20Spelke\%202010.pdf, 61(2). https://doi.org/10.1016/j.cogpsych.2010.04.002

Lever, C., Wills, T., Cacucci, F., Burgess, N., \& O'Keefe, J. (2002). Long-term plasticity in hippocampal place-cell representation of environmental geometry. Nature, 416(6876), 90-94. https://doi.org/10.1038/416090a

Lew, A. R., Gibbons, B., Murphy, C., \& Gavin Bremner, J. (2010). Use of geometry for spatial reorientation in children applies only to symmetric spaces. Developmental Science, 13(3), 490-498. https://doi.org/10.1111/j.14677687.2009.00904.X

Li, R., \& Klippel, A. (2016). Wayfinding Behaviors in Complex Buildings: The Impact of Environmental Legibility and Familiarity [research-article]. Environment and Behavior, 48(3), 482-510. https://doi.org/10.1177 0013916514550243

Liang, M., Starrett, M. J., \& Ekstrom, A. D. (2018). Dissociation of frontal-midline delta-theta and posterior alpha oscillations: A mobile EEG study. Psychophysiology, 55(9), n/a-n/a. https://doi.org/10.1111/psyp.13090

Lin, C. T., Chiu, T. C., \& Gramann, K. (2015). EEG correlates of spatial orientation in the human retrosplenial complex. Neuroimage, 120, 123-132. https://doi.org/10.1016/j.neuroimage.2015.07.009

Long, N. M., Burke, J. F., \& Kahana, M. J. (2014). Subsequent memory effect in intracranial and scalp EEG. NeuroImage (Orlando, Fla.), 84, 488-494. https://doi.org/10.1016/j.neuroimage.2013.08.052

Luo, J., \& Niki, K. (2003). Function of hippocampus in "insight" of problem solving. Hippocampus, 13(3), 316-323. https://doi.org/10.1002/hipo.10069

Luo, J., Niki, K., \& Knoblich, G. (2006). Perceptual contributions to problem solving: Chunk decomposition of Chinese characters. Brain research bulletin, 70(4-6), 430-443. https://doi.org/10.1016/j.brainresbull.2006.07.005 
Maguire, E. A., Burgess, N., Donnett, J. G., Frackowiak, R. S., Frith, C. D., \& Keefe, J. (1998). Knowing where and getting there: a human navigation network. Science (New York, N.Y.), 280(5365), 921.

Mai, X. Q., Luo, J., Wu, J. H., \& Luo, Y. J. (2004). "Aha!" effects in a guessing riddle task: an event-related potential study. Human brain mapping, 22(4), 261-270. https://doi.org/10.1002/hbm.20030

Marchette, S. A., Vass, L. K., Ryan, J., \& Epstein, R. A. (2014). Anchoring the neural compass: coding of local spatial reference frames in human medial parietal lobe. Nature Neuroscience, 17(11). https://doi.org/10.1038/nn.3834

Massumi, B. (1999). Strange Horizon: Buildings, Biograms, and the Body Topologic [Journal article]. Architectural Design, 12-19. https://doi.org/http://hdl.handle.net/1885/91610

Meilinger, T. (2008). Strategies of orientation in environmental spaces. Logos Verlag Berlin GmbH.

Meilinger, T., Riecke, B. E., \& Bülthoff, H. H. (2014). Local and Global Reference Frames for Environmental Spaces. Quarterly Journal of Experimental Psychology, 67(3), 542-569. https://doi.org/10.1080/17470218.2013.821145

Melnik, A., Legkov, P., Izdebski, K., Kärcher, S. M., Hairston, W. D., Ferris, D. P., \& König, P. (2017). Systems, Subjects, Sessions: To What Extent Do These Factors Influence EEG Data? Frontiers in human neuroscience, 11, 150. https://doi.org/10.3389/fnhum.2017.00150

Micah, A., Davide, P., Kirstie, W., Tom Rhys, M., \& Rogier, A. K. (2019). Raincloud plots: a multi-platform tool for robust data visualization [version 1; peer review: 2 approved]. Wellcome Open Research, 4. https://doi.org/10.12688/wellcomeopenres.15191.1

Milivojevic, B., Vicente-Grabovetsky, A., \& Doeller, C. f. (2015). Insight Reconfigures Hippocampal-Prefrontal Memories. Current Biology, 25(7), 821-830. https://doi.org/10.1016/j.cub.2015.01.033

Mitchell, D. J., McNaughton, N., Flanagan, D., \& Kirk, I. J. (2008). Frontal-midline theta from the perspective of hippocampal "theta". Progress in Neurobiology, 86(3), 156-185. https://doi.org/10.1016/j.pneurobio.2008.09.005

Montello, D. R. (1998). A new framework for understanding the acquisition of spatial knowledge in large-scale environments. In M. J. Egenhofer \& R. G. Golledge (Eds.), Spatial and temporal reasoning in geographic information systems (pp. 143-154). New York: Oxford University Press.

Montello, D. R. (2007). The contribution of space syntax to a comprehensive theory of environmental psychology. Proceedings of the 6th International Space Syntax Symposium, Istanbul.

Montello, D. R. (2009). Geographic Orientation \& Disorientation: Getting Lost and Getting Found in Real and Information Spaces. User Experience Magazine, $8(1), 24-26$.

Montello, D. R. (2020). Geographic orientation, disorientation, and misorientation: a commentary on Fernandez Velasco and Casati. Spatial Cognition \& Computation, 1-8. https://doi.org/10.1080/13875868.2020.1767105

Mou, W., McNamara, T. P., Rump, B., \& Xiao, C. (2006). Roles of Egocentric and Allocentric Spatial Representations in Locomotion and Reorientation. Journal of Experimental Psychology: Learning, Memory, and Cognition, 32(6), 1274-1290. https://doi.org/10.1037/0278-7393.32.6.1274

Newcombe, N. S., Ratliff, K. R., Shallcross, W. L., \& Twyman, A. D. (2010). Young children's use of features to reorient is more than just associative: further 
evidence against a modular view of spatial processing. Developmental Science, 13(1), 213-220. https://doi.org/10.1111/j.1467-7687.2009.00877.x

Pagkratidou, M., Galati, A., \& Avraamides, M. (2020). Do environmental characteristics predict spatial memory about unfamiliar environments? Spatial Cognition \& Computation, 20(1), 1-32. https://doi.org/10.1080/13875868.2019.1676248

Patai, E., Marin-Garcia, E., Margolis, A., Tan, H.-R., Kumaran, D., Nardini, M., Will, P., Duzel, E., Dayan, P., \& Spiers, H. (2019). Prefrontal Dynamics Associated with Efficient Detours and Shortcuts: A Combined Functional Magnetic Resonance Imaging and Magnetoencenphalography Study. Journal of Cognitive Neuroscience, 31(8), 1227-1247. https://doi.org/10.1162/jocn_a 01414

Penn, A. (2003). Space syntax and spatial cognition: or why the axial line? Environment and behavior, 35(1), 30-65.

Peponis, J., Zimring, C., \& Choi, Y. K. (1990). Finding the Building in Wayfinding. Environment and Behavior, 22(5), 555-590. https://doi.org/10.1177/0013916590225001

Pietto, M., Raimondo, F., \& Kamienkowski, J. (2018). Electrophysiological approaches in the study of cognitive development outside the lab. PLoS One, 13(11), e0206983. https://doi.org/10.1371/journal.pone.0206983

Pisula, W., Modlinska, K., \& Chrzanowska, A. (2019). Behavioural Response to the Environmental Changes of Various Types in Lister-Hooded Male Rats. Scientific reports, 9(1), 7111. https://doi.org/10.1038/s41598-019-42924-1

Qiu, J., Li, H., Luo, Y., Chen, A., Zhang, F., Zhang, J., Yang, J., \& Zhang, Q. (2006). Brain mechanism of cognitive conflict in a guessing Chinese logogriph task. NeuroReport, 17(6), 679-682.

Rünger, D., \& Frensch, P. A. (2008). How incidental sequence learning creates reportable knowledge: the role of unexpected events. J Exp Psychol Learn Mem Cogn, 34(5), 1011-1026. https://doi.org/10.1037/a0012942

Salvi, C., Beeman, M., Bikson, M., Mckinley, R., \& Grafman, J. (2020). TDCS to the right anterior temporal lobe facilitates insight problem-solving. Scientific Reports, 10(1), 1-10. https://doi.org/10.1038/s41598-020-57724-1

Sandkuhler, S., \& Bhattacharya, J. (2008). Deconstructing insight: EEG correlates of insightful problem solving. PLoS One, 3(1), e1459. https://doi.org/10.1371/journal.pone.0001459

Schacter, D. L., Addis, D. R., \& Buckner, R. L. (2007). Remembering the past to imagine the future: the prospective brain. Nature Reviews Neuroscience, 8(9), 657. https://doi.org/10.1038/nrn2213

Shen, W., Luo, J., Liu, C., \& Yuan, Y. (2013). New advances in the neural correlates of insight: A decade in review of the insightful brain. Chinese Science Bulletin, 58(13), 1497-1511. https://doi.org/10.1007/s11434-012-5565-5

Shen, W., Tong, Y., Li, F., Yuan, Y., Hommel, B., Liu, C., \& Luo, J. (2018). Tracking the neurodynamics of insight: A meta-analysis of neuroimaging studies. Biological Psychology, 138, 189-198. https://doi.org/10.1016/j.biopsycho.2018.08.018

Shen, W., Yuan, Y., Liu, C., \& Luo, J. (2017). The roles of the temporal lobe in creative insight: an integrated review. Thinking \& Reasoning, 23(4), 321-375. https://doi.org/10.1080/13546783.2017.1308885

Snider, J., Plank, M., Lynch, G., Halgren, E., \& Poizner, H. (2013). Human cortical $\theta$ during free exploration encodes space and predicts subsequent memory. The 
Journal of neuroscience, 33(38), 15056-15068.

https://doi.org/10.1523/JNEUROSCI.0268-13.2013

Spaak, E., \& de Lange, F. P. (2020). Hippocampal and Prefrontal Theta-Band Mechanisms Underpin Implicit Spatial Context Learning. The Journal of neuroscience, 40(1), 191-202. https://doi.org/10.1523/jneurosci.1660-19.2019

Stachenfeld, K. L., Botvinick, M. M., \& Gershman, S. J. (2017). The hippocampus as a predictive map. Nature Neuroscience, 20(11), 1643. https://doi.org/10.1038/nn.4650

Stamps, A. E. (2008). On Shape and Spaciousness. Environment and Behavior, 41(4), 526-548. https://doi.org/10.1177/0013916508317931

Stamps, A. E. (2011). Effects of Area, Height, Elongation, and Color on Perceived Spaciousness. Environment and Behavior, 43(2), 252-273. https://doi.org/10.1177/0013916509354696

Staudigl, T., \& Hanslmayr, S. (2013). Theta Oscillations at Encoding Mediate the Context-Dependent Nature of Human Episodic Memory. Current biology, 23(12), 1101-1106. https://doi.org/10.1016/j.cub.2013.04.074

Strange, B. A., Duggins, A., Penny, W., Dolan, R. J., \& Friston, K. J. (2005). Information theory, novelty and hippocampal responses: unpredicted or unpredictable? Neural Networks, 18(3), 225-230. https://doi.org/10.1016/j.neunet.2004.12.004

Sturz, B. R., Gurley, T., \& Bodily, K. D. (2011). Orientation in Trapezoid-Shaped Enclosures: Implications for Theoretical Accounts of Geometry Learning. Journal of Experimental Psychology: Animal Behavior Processes, 37(2), 246253. https://doi.org/10.1037/a0021215

Turner, A. (2001). Depthmap: A Program to Perform Visibility Graph Analysis. the Third International Space Syntax Symposium, Georgia Technological Institute.

Turner, A., Doxa, M., O'Sullivan, D., \& Penn, A. (2001). From isovists to visibility graphs: a methodology for the analysis of architectural space. Environment and Planning B: Planning \& Design, 28.

Vann, S. D., Aggleton, J. P., \& Maguire, E. A. (2009). What does the retrosplenial cortex do? Nature Reviews Neuroscience, 10(11), 792. https://doi.org/10.1038/nrn2733

Vitz, P. C. (1966). Preference for different amounts of visual complexity. Behavioral Science, 11(2), 105-114. https://doi.org/10.1002/bs.3830110204

Watrous, A. J., Fried, I., \& Ekstrom, A. D. (2011). Behavioral correlates of human hippocampal delta and theta oscillations during navigation. Journal of neurophysiology, 105(4), 1747. https://doi.org/10.1152/jn.00921.2010

Weisberg, R. W. (2013). On the "Demystification" of Insight: A Critique of Neuroimaging Studies of Insight. Creativity Research Journal, 25(1), 1-14. https://doi.org/10.1080/10400419.2013.752178

Wills, T. J., Lever, C., Cacucci, F., Burgess, N., \& O'Keefe, J. (2005). Attractor dynamics in the hippocampal representation of the local environment. Science (New York, N.Y.), 308(5723), 873.

Wolbers, T., \& Wiener, J. M. (2014). Challenges for identifying the neural mechanisms that support spatial navigation: the impact of spatial scale. Front Hum Neurosci, 8, 571. https://doi.org/10.3389/fnhum.2014.00571

Zhao, Q., Zhou, Z., Xu, H., Chen, S., Xu, F., Fan, W., \& Han, L. (2013). Dynamic neural network of insight: a functional magnetic resonance imaging study on solving Chinese 'chengyu' riddles. PLoS ONE, 8(3), e59351. https://doi.org/10.1371/journal.pone.0059351 
Zimring, C., \& Dalton, R. C. (2003). Linking Objective Measures Of Space To Cognition And Action. Environment and Behavior, 35(1), 3-16.

https://doi.org/10.1177/0013916502238862 